\title{
Wnt5a Mediates Nerve Growth Factor-Dependent Axonal Branching and Growth in Developing Sympathetic Neurons
}

\author{
Daniel Bodmer, ${ }^{\star}$ Seamus Levine-Wilkinson,${ }^{\star}$ Alissa Richmond, Sarah Hirsh, and Rejji Kuruvilla \\ Department of Biology, The Johns Hopkins University, Baltimore, Maryland 21218
}

Nerve growth factor (NGF) is a potent survival and axon growth factor for neuronal populations in the peripheral nervous system. Although the mechanisms by which target-derived NGF influences survival of innervating neurons have been extensively investigated, its regulation of axonal growth and target innervation are just being elucidated. Here, we identify Wnt5a, a member of the Wnt family of secreted growth factors, as a key downstream effector of NGF in mediating axonal branching and growth in developing sympathetic neurons. Wnt5a is robustly expressed in sympathetic neurons when their axons are innervating NGF-expressing targets. NGF:TrkA signaling enhances neuronal expression of Wnt5a. Wnt5a rapidly induces axon branching while it has a long-term effect on promoting axon extension. Loss of Wnt5a function revealed that it is necessary for NGF-dependent axonal branching and growth, but not survival, in vitro. Furthermore, Wnt5a $a^{-1-}$ mice display reduced innervation of NGF-expressing target tissues, and a subsequent increase in neuronal apoptosis, in vivo. Wnt5a functions in developing sympathetic neurons by locally activating protein kinase $\mathrm{C}$ in axons. Together, our findings define a novel regulatory pathway in which Wnt5a, expressed in sympathetic neurons in response to target-derived NGF, regulates innervation of peripheral targets.

\section{Introduction}

To form functional connections during neuronal development, neurons send out axonal projections that navigate through a series of intermediate targets to reach their final target tissues. Axon growth en route to targets encompasses elongation, fasciculation/ defasciculation, changes in axon caliber, interstitial branching to make connections with intermediate targets, and extensive terminal branching to innervate final target fields (Rubin, 1985). This latter feature of developing axons is critical for synapse formation and establishment of functional neuronal circuits. Target-derived growth factors are known to influence the density of target innervation (Edwards et al., 1989; Diamond et al., 1992; Causing et al., 1997). However, the precise signaling mechanisms by which target-derived trophic factors regulate axonal arborization and innervation of final target fields are poorly understood.

The neurotrophin, nerve growth factor (NGF), is a targetderived signal that regulates terminal arborization in developing sympathetic and sensory neurons in the peripheral nervous system (Patel et al., 2000; Glebova and Ginty, 2004; Kuruvilla et al., 2004;). Overexpression of NGF in target tissues enhanced growth of sympathetic and sensory nerves into the targets (Edwards et al., 1989; Albers et al., 1994; Hassankhani et al., 1995). Conversely,

Received March 26, 2009; accepted May 6, 2009.

This work was supported with funds from National Institutes of Health Grant R01 MH080738 and a Whitehall Foundation award (R.K.). We thank Andreas Kispert for providing the Wnt5a expression vector, Chen-ming Fan for the dominant-negative TCF adenovirus, and David Ginty for the TrkA ${ }^{\text {F592A }}$ mice and 1NMPP1. We are grateful to Haiqing Zhao, Samer Hattar, and members of the Kuruvilla Laboratory for comments on this manuscript and helpful discussions.

*D.B. and S.L.-W. contributed equally to this work.

Correspondence should be addressed to Rejji Kuruvilla, Department of Biology, The Johns Hopkins University, Baltimore, MD 21218. E-mail: rkuruvilla@jhu.edu.

DOI:10.1523/JNEUROSCI.1445-09.2009

Copyright $\odot 2009$ Society for Neuroscience $\quad 0270-6474 / 09 / 297569-13 \$ 15.00 / 0$ sympathetic and sensory innervation of final target fields is either absent or incomplete in mice lacking NGF or its cognate receptor, TrkA (Patel et al., 2000; Glebova and Ginty, 2004; Kuruvilla et al., 2004). Interestingly, the initial phases of axonal outgrowth from peripheral ganglia and extension along intermediate targets seem to be NGF independent (Fagan et al., 1996; Glebova and Ginty, 2004; Wickramasinghe et al., 2008). These studies suggest that target-derived NGF is critical for axonal extension and arborization only after the axons have reached their final destinations. NGF promotes a wide spectrum of actions on peripheral axons in vitro including elongation, increases in caliber, branching, growth cone turning responses, and changes in growth cone morphology (Gallo and Letourneau, 1998; Markus et al., 2002), all of which may be required for NGF-mediated innervation of final target tissues in vivo.

In addition to target-derived NGF, recent studies indicate that Wnts secreted by target tissues act as potent axonal branching and synaptogenic factors to influence target innervation (Ciani and Salinas, 2005). Wnt7a expressed by cerebellar granule cells induces growth cone enlargement and axonal spreading of presynaptic mossy fibers (Lucas and Salinas, 1997; Hall et al., 2000). Wnt3 secreted by motoneurons regulates axonal branching and terminal arborization of NT-3-responsive spinal sensory neurons (Krylova et al., 2002).

Here, we present evidence that NGF promotes axonal branching and target innervation in developing sympathetic targets by regulating the neuronal expression of Wnt5a. Wnt5a is most robustly expressed at a developmental time when axons are reaching final targets. NGF stimulation of sympathetic neurons enhanced levels of Wnt5a transcript and protein. Wnt5a had an acute effect on inducing axonal branching and a long-term effect on enhancing axon extension. Sympathetic neurons derived 
from Wnt5a-null mice show deficits in NGF-dependent axonal branching and growth, but not survival, in vitro. Analysis of $W n t 5 a^{-l-}$ mice revealed abnormalities in sympathetic chain formation and reduced innervation of target tissues, which lead to enhanced cell death later in development. Wnt5a functions in sympathetic neurons by activating a signaling pathway that is independent of transcription and requires protein kinase $\mathrm{C}$ (PKC) locally in axons. Thus, interactions between Wnt5a expressed in sympathetic neurons and NGF secreted by neuronal targets regulate growth events required for innervation.

\section{Materials and Methods}

Transgenic animals. Wnt5a ${ }^{+/-}$and TOPGAL reporter mice were obtained from The Jackson Laboratory. The generation of TrkA ${ }^{\text {F592A }}$ mice has been previously described (Chen et al., 2005). All procedures relating to animal care and treatment conformed to institutional and National Institutes of Health guidelines.

In situ hybridization. In situ hybridization was done using digoxigenin (DIG)-labeled cRNA probe specific for mouse Wnt5a. The probe was generated from a pGEM-7zf $(+)$ plasmid containing a $2.5 \mathrm{~kb}$ insert with the entire coding region of mouse Wnt5a. Embryos were fixed overnight in $4 \%$ paraformaldehyde (PFA) (in $0.1 \mathrm{M} \mathrm{PBS}$ ), whereas postnatal pups were transcardially perfused with $4 \%$ PFA and postfixed overnight at $4^{\circ} \mathrm{C}$. After cryoprotection overnight with $20 \%$ sucrose (in PBS) and embedding in OCT (Tissue-Tek), serial cryostat sections $(14 \mu \mathrm{m})$ were prepared and mounted on SuperFrost Plus slides (Thermo Fisher Scientific). Tissue sections were permeabilized with $0.1 \%$ Triton X-100 (in 0.1 M PBS) for $15 \mathrm{~min}$ at room temperature and then acetylated with $0.25 \%$ acetic anhydride in $0.1 \mathrm{~m}$ triethanolamine with $0.9 \% \mathrm{NaCl}$. Sections were hybridized overnight with labeled RNA probe $(1 \mu \mathrm{g} / \mathrm{ml})$ at $65^{\circ} \mathrm{C}$, washed in $2 \times$ SSC buffer at $60^{\circ} \mathrm{C}$, blocked with TBS containing $1 \%$ blocking reagent (Roche), and then incubated with alkaline phosphatase-labeled anti-DIG antibody (1:3000; Roche) overnight. Sections were washed and the alkaline phosphatase reaction visualized with NBT/BCIP (nitroblue tetrazolium/5-bromo-4-chloro-3-indolyl phosphate) (Roche) followed by rinsing in PBS, fixation in 4\% PFA, and mounting the slides using VectaMount AQ (Vector Laboratories).

Immunohistochemical analyses. Tissue sections $(16 \mu \mathrm{m})$ were rinsed in PBS followed by a 15 min fixation in ice-cold $4 \%$ paraformaldehyde in PBS. Sections were then permeabilized in 1\% Triton X-100 in PBS for 15 min, followed by blocking $(0.5 \%$ Triton X-PBS, $5 \%$ goat serum; $1 \mathrm{~h})$ at room temperature. For immunofluorescence, mouse anti-tyrosine hydroxylase (TH-16 ascites; 1:100; Sigma-Aldrich) was applied to sections overnight at $4^{\circ} \mathrm{C}$ in a dark, humidified chamber. Sections were then incubated with Alexa 488-conjugated antibody to mouse $\operatorname{IgG}(\gamma 1)(1: 200$; Invitrogen). Sections were then washed and mounted in Vectashield (Vector Laboratories). For colorimetric readout, rabbit anti-TH (1:200; Millipore Bioscience Research Reagents) was applied to sections overnight at $4^{\circ} \mathrm{C}$, washed, incubated with HRP conjugated to rabbit IgG (1:500; GE Healthcare), and immunoreactivity was detected with diaminobenzidine (Sigma-Aldrich). Whole-mount TH immunostaining to visualize sympathetic chains and axons was performed in embryonic day 16.5 (E16.5) mouse embryos as described previously (Glebova and Ginty, 2004). For $\beta$-galactosidase histochemistry, tissue sections (10 $\mu \mathrm{m})$ were incubated overnight at room temperature in freshly prepared X-gal staining solution $\left(2 \mathrm{mM} \mathrm{MgCl}_{2}, 0.01 \%\right.$ sodium deoxycholate, $0.02 \%$ IGEPAL, $5 \mathrm{~mm}$ potassium ferricyanide, $5 \mathrm{~mm}$ potassium ferrocyanide, and 1 $\mathrm{mg} / \mathrm{ml} \mathrm{X}$-gal). After X-gal staining, sections were rinsed in PBS and fixed for $10 \mathrm{~min}$ in $4 \%$ PFA-PBS. Sections were then counterstained with Nuclear Fast Red (Vector Laboratories) for 3-5 min. Counterstain reaction was stopped with 10 min treatment of $70 \%$ ethanol, and sections were mounted on slides with $100 \%$ glycerol.

Neuronal cell counts. Neuronal cell counts in SCGs were performed as previously described (Chen et al., 2005). Heads of E13.5, E15.5, E17.5, and postnatal day 0.5 (P0.5) mice were fixed in PBS containing 4\% PFA, and then cryoprotected in 30\% sucrose-PBS, overnight. SCG sections $(10 \mu \mathrm{m})$ were stained with a solution containing $0.5 \%$ cresyl violet (Nissl). Cells with characteristic neuronal morphology and visible nucle- oli were counted in every fifth Nissl stained section. For E13.5, E15.5, and E17.5 embryos, sections were also stained for $\mathrm{TH}$, and immunopositive cells were counted in every fifth section.

PCR analyses. For real-time quantitative PCR (qPCR) analyses, total RNA was prepared from mouse sympathetic neuron cultures using the RNeasy Micro Kit (QIAGEN). Poly(A) RNAs were reverse transcribed using RETROscript kit (Ambion). Real-time qPCR was performed with iQ SYBR Green Supermix and iCycler iQ real-time PCR detection system (Bio-Rad). Each sample was analyzed in four replicate reactions of $50 \mu \mathrm{l}$.

Immunocytochemistry, immunoblotting, and antibodies. Cells were fixed with 4\% PFA in PBS, blocked with 3\% BSA in PBS, and immunostained overnight with primary antibodies against Wnt5a (1:1000; Neuromics or R\&D Systems) or $\beta$-III-tubulin (1:500; Sigma-Aldrich). Images were acquired using either confocal microscopy (Zeiss LSM510 Meta confocal microscope) or epifluorescence microscopy (Zeiss Axioplan microscope). After washing, neurons were incubated with appropriate secondary antibodies (Alexa Fluor; Invitrogen). Immunoblotting analyses of sympathetic neuron lysates were performed as described previously (Kuruvilla et al., 2004). For NGF-dependent regulation of Wnt5a protein, sympathetic neurons were grown for $4-5 \mathrm{~d}$ in vitro in the presence of NGF $(50 \mathrm{ng} / \mathrm{ml})$ and $\mathrm{AraC}(10 \mu \mathrm{M})$, and then starved of NGF in the presence of boc-aspartyl(OMe)-fluoromethylketone (BAF) for $2 \mathrm{~d}$ and then stimulated with NGF $(50 \mathrm{ng} / \mathrm{ml})$ for different time points before being harvested for Wnt5a immunoblotting. For sympathetic neurons from TrkA ${ }^{\mathrm{F} 592 \mathrm{~A}}$ mice, SCG neurons from P0.5 mice were cultured in the presence of NGF (50 ng/ml) and AraC (10 $\mu \mathrm{M})$ for $4-5 \mathrm{~d}$ in vitro, then treated with 4-amino-1-tert-butyl-3-(1' -naphthylmethyl)pyrazolo[3,4d] pyrimidine (1NMPP1) (100 nM; 12 h), and lysates harvested for Wnt5a immunoblotting. The following antibodies were used for immunoblotting: Wnt5a (R\&D Systems), $\alpha$-tubulin (Sigma-Aldrich), and p85 subunit of PI-3 kinase (Millipore). All phosphospecific antibodies including p-PKC (pan, $\beta$ II Ser660), p-c-Jun (Ser63), p-calcium-calmodulin kinase II (p-CaMKII) (Thr286), p-GSK-3 $\beta$ (Ser9), p-Akt (Ser473), and p-Erk1/2 (Thr202/Tyr204) were obtained from Cell Signaling Technology.

Neuronal cultures and quantification of neurite length, axon branching, and neuronal survival. SCGs, dissected from wild-type and Wnt5a ${ }^{-1-}$ embryos (E18.5) obtained from Wnt5a heterozygous mothers, were cultured in collagen gel in media containing NGF $(50 \mathrm{ng} / \mathrm{ml})$ for $72 \mathrm{~h}$, and then immunostained with $\beta$-III-tubulin. For rescue experiments with exogenous Wnt5a, E16.5 Wnt5a ${ }^{-1-}$ SCG explants were grown for $72 \mathrm{~h}$ in collagen gel in control or Wnt5a conditioned media supplemented with NGF $(50 \mathrm{ng} / \mathrm{ml})$, and then immunostained for $\beta$-III-tubulin. Neurite outgrowth in SCG explants was quantified by measuring the area covered by the axons of each explant relative to the area occupied by the cell bodies. Low-density cultures were established from P0.5 rat pups or E18.5 wild-type and $W n t 5 a^{-1-}$ embryos and cultured on polylysine/ laminin-coated coverslips (for 8 or $24 \mathrm{~h}$ as indicated in the figure legends). To visualize neuronal morphology in fixed cells, neurons were stained with $\beta$-III-tubulin antibody. To measure neurite length and axonal branching of isolated sympathetic neurons, random neurons (at least 50 clearly separated neurons selected per dish) were imaged using a Zeiss Axiovision microscope with a AxioCam HRC digital camera and analyzed with Axiovision software. Length (in micrometers) of the longest neurite as well as the total number of branch points were quantified for each neuron. Neurites longer than $5 \mu \mathrm{m}$ were counted as branches.

To determine the effects of exogenous Wnt $5 \mathrm{a}$ on sympathetic neuron survival, dissociated neurons from $\mathrm{P} 0.5$ rat pups were cultured in the presence of NGF $(10 \mathrm{ng} / \mathrm{ml})$ for $4-5 \mathrm{~d}$ in vitro to allow elaboration of a dense network of axons. Neurons were then washed to remove NGF and cultured for another $2 \mathrm{~d}$ in Wnt5a or control conditioned media in the presence or absence of NGF $(10 \mathrm{ng} / \mathrm{ml})$. Cultures grown in the absence of NGF were treated with a neutralizing antibody to NGF (anti-NGF; 1:1000; Sigma-Aldrich) to ensure complete removal of NGF from the culture media. To assess the ability of wild-type and Wnt5a $a^{-1-}$ neurons to survive in the presence of NGF, sympathetic neurons harvested from E15.5 wild-type and $W n t 5 a^{-1-}$ embryos were cultured for $4-5 \mathrm{~d}$ in vitro in different concentrations of NGF $(1,10$, and $30 \mathrm{ng} / \mathrm{ml})$-containing media and then stained with the nuclear dye, Hoechst 33258 (Invitro- 
gen). Neurons with pyknotic, condensed, or fragmented nuclei were scored as dead.

To block transcription, neurons were plated and cultured for $8 \mathrm{~h}$ in control conditioned medium and then treated with control or Wnt5a conditioned medium in the presence or absence of actinomycin D $(0.1 \mu \mathrm{g} / \mathrm{ml})$ for $16 \mathrm{~h}$. Longer incubations with actinomycin D resulted in cytotoxic effects. To block translation, neurons were cultured overnight in control conditioned medium, pretreated with cycloheximide $(1 \mu \mathrm{g} / \mathrm{ml}$ ) or vehicle (DMSO) for $15 \mathrm{~min}$, and then treated with control or Wnt5a conditioned medium in the presence or absence of cycloheximide $(1 \mu \mathrm{g} / \mathrm{ml})$ for $1 \mathrm{~h}$. To investigate the requirement of PKC in Wnt5a-dependent axonal branching, neurons were cultured in control or Wnt5a conditioned media in the presence or absence of myristoylated PKC $\alpha$ pseudosubstrate (25 $\mu \mathrm{M}$; catalog \#P-205; BIOMOL) for $24 \mathrm{~h}$.

Live imaging. Sympathetic neurons cultured for $8 \mathrm{~h}$ in control conditioned media were treated with control or Wnt5a conditioned medium, and images taken at $30 \mathrm{~min}$ intervals for up to $2 \mathrm{~h}$. Neurons were viewed with a $20 \times / 0.4$ Neofluar phase contrast objective attached to a Zeiss Axiovert microscope, and images were taken with a Retiga Exi digital camera with Openlab software (Improvision).

Axonal extension and branching in compartmentalized cultures. Compartmentalized cultures of sympathetic neurons from $\mathrm{P} 0.5$ rat pups were set up, and axonal elongation (in micrometers per day) was measured as described previously (Ye et al., 2003). Compartmentalized cultures were initially maintained in NGF-containing media for 5-7 d to allow robust axonal growth into axonal compartments. To analyze Wnt-mediated effects alone, NGF was withdrawn from the culture media bathing cell bodies and axons on addition of either control or Wnt5a media to axonal compartments. Distal axon compartments were photographed every $24 \mathrm{~h}$ for the next $3 \mathrm{~d}$ using a Zeiss Axiovert microscope equipped with a Retiga EXi camera. Axon length was measured using Openlab software (Improvision) and expressed as average axon growth per day (in micrometers per day). For axon branching, low-density sympathetic cultures in compartmentalized chambers were established to allow visualization of "single" axons projecting into side compartments. In all axon growth and branching assays, the caspase inhibitor BAF $(50 \mu \mathrm{M})$ was added to cultured neurons to prevent cell death in absence of NGF. In experiments neutralizing Wnt5a, axons in compartmentalized cultures were treated with media containing anti-NGF (1:1000), NGF $(50 \mathrm{ng} / \mathrm{ml})$, or NGF $(50 \mathrm{ng} / \mathrm{ml})$ plus anti-Wnt $5 \mathrm{a}(5 \mu \mathrm{g} / \mathrm{ml})$ for $72 \mathrm{~h}$. For axons treated with anti-NGF or NGF alone, goat IgG $(5 \mu \mathrm{g} / \mathrm{ml})$ was included in the media as a control. In all experimental conditions, cell bodies were maintained in media containing anti-NGF. Neurons were fixed and immunostained for $\beta$-III-tubulin (1:200 dilution; Sigma-Aldrich). Axonal branching was measured by photographing axonal compartments using a Zeiss Axiovert microscope equipped with a Retiga EXi camera, and counting the total number of axonal branch points for all axons per collagen track. For every axon, each point of divergence from the main axonal shaft was counted as a branch point. For axonal branching assays involving $\mathrm{PKC} \alpha$ pseudosubstrate, compartmentalized cultures were treated with control or Wnt5a-containing media on distal axons in the presence or absence of PKC $\alpha$ pseudosubstrate $(25 \mu \mathrm{M})$ on cell bodies or distal axons. Quantification of axonal branch points was performed by photographing axons at 0 and $24 \mathrm{~h}$ after treatment and counting the number of new branch points formed per projection.

Statistical analyses. Statistical comparisons were determined by the Student's $t$ test for pair comparisons and by one-way ANOVA for multiple comparisons. Post hoc analyses were done using Tukey-Kramer's test. Values and error bars indicate mean \pm SEM.

\section{Results}

\section{NGF regulates Wnt5a expression in sympathetic neurons}

Levels of $W n t 5 a$ transcript, as detected by in situ hybridization, are low in the primordial SCG at E13.5 (Fig. 1A), a developmental stage when the SCG is coalescing into a discrete ganglion. Robust expression of Wnt5a in the SCG was observed at E16.5 (Fig. $1 B$ ) and P0.5 (Fig. 1C), when there is peak innervation of sympathetic targets. Sense control showed little staining indicating the specificity of the in situ probe (supplemental Fig. 1A, $B$, available at www.jneurosci.org as supplemental material). By P14.5 (Fig. 1D) and in the adult SCG at P40.5 (Fig. 1E), Wnt5a expression is restricted to a subpopulation of neurons. Immunocytochemical analyses in dissociated SCG cultures showed that the Wnt5a protein is localized primarily to sympathetic neurons and not residual glial cells (supplemental Fig. $1 C, D$, available at www.jneurosci.org as supplemental material). Together, these results indicate that $\mathrm{Wnt} 5 \mathrm{a}$ expression in developing sympathetic neurons is synchronous with the timing of target field innervation.

Given the prominent expression of Wnt5a in sympathetic neurons at developmental times when sympathetic axons are being exposed to target-derived NGF in vivo, we asked whether NGF regulates Wnt5a expression. Sympathetic neurons isolated from mouse P0.5 SCGs were cultured for 12, 24, and $48 \mathrm{~h}$ in the presence or absence of NGF. The broad-spectrum caspase inhibitor BAF was added to the cell cultures to circumvent neuronal apoptosis in the absence of NGF. Wnt5a transcript levels were assayed by quantitative real-time PCR analyses. NGF treatment significantly enhanced Wnt5a mRNA levels in cultured sympathetic neurons at all three time points examined (Fig. $1 F)(12 \mathrm{~h}$, $p=0.0068 ; 24 \mathrm{~h}, p=0.0075$; and $48 \mathrm{~h}, p=0.0412$, two-tailed Student's $t$ test), whereas expression of Wnt4 and Wnt11 were unaffected by NGF (supplemental Fig. $2 A$, available at www.jneurosci.org as supplemental material). We also cultured sympathetic neurons for $5 \mathrm{~d}$ in vitro in the continued presence of NGF $(50 \mathrm{ng} / \mathrm{ml})$ to allow the formation of a dense network of axons, after which the neurons were starved for $2 \mathrm{~d}$ in the presence of BAF, and then either left untreated or stimulated with NGF ( $50 \mathrm{ng} / \mathrm{ml}$ ) for $12 \mathrm{~h}$. We observed a similar twofold elevation of Wnt5a transcript levels with NGF treatment (supplemental Fig. $2 B$, available at www.jneurosci.org as supplemental material) as seen in Figure $1 F$, providing more definitive evidence that upregulation of $W n t 5 a$ is the result of NGF stimulation rather than being secondary to a global trophic effect of NGF. In addition, Wnt5a levels were not influenced by treatment of sympathetic neurons with NT-3 (Fig. 1G), a neurotrophin highly expressed in blood vessels along the trajectory of sympathetic axons (Francis et al., 1999) and that promotes robust neurite outgrowth by activating TrkA receptors (Belliveau et al., 1997; Kuruvilla et al., 2004). NGF treatment also increased amounts of Wnt5a protein in cultured sympathetic neurons (Fig. $1 H$ ).

To further address the role of NGF in regulating Wnt5a expression, we used TrkA ${ }^{\text {F592A }}$ mice, a knock-in mouse line expressing the NGF receptor, TrkA, with a mutated ATP binding pocket (Chen et al., 2005). With this modification, TrkA receptors can be selectively and reversibly inhibited by the smallmolecule membrane-permeable inhibitor 1NMPP1 (Chen et al., 2005). In sympathetic neuron cultures established from TrkA ${ }^{\text {F592A }}$ mice, we found that inhibition of TrkA kinase activity with 1NMPP1 substantially attenuated Wnt5a levels in neurons maintained in the presence of NGF (Fig. 1I) (percentage decrease of $35 \pm 5.6 \% ; p=0.0038$, paired, two-tailed Student's $t$ test; $n=$ 4 independent experiments). 1NMPP1 had no effect on Wnt5a expression in neurons expressing wild-type unmodified TrkA receptors (supplemental Fig. 3, available at www.jneurosci.org as supplemental material). These results suggest that Wnt5a expression in sympathetic neurons is dependent on NGF:TrkA signaling. 
A

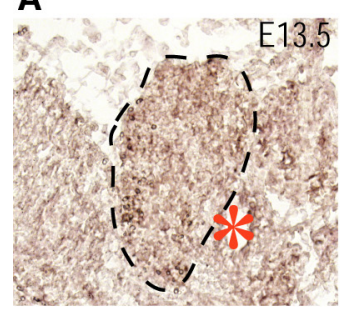

F

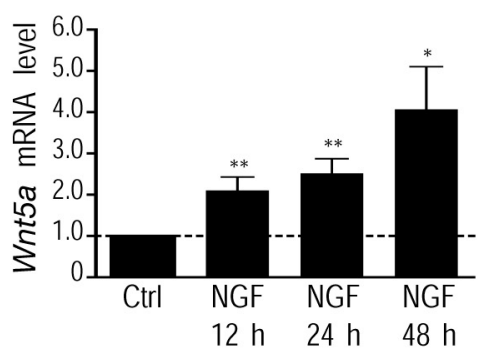

B

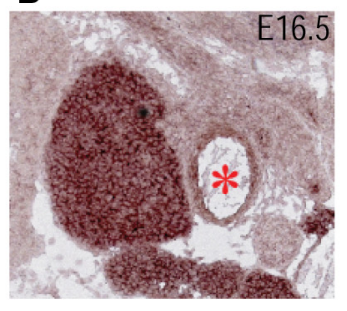

G

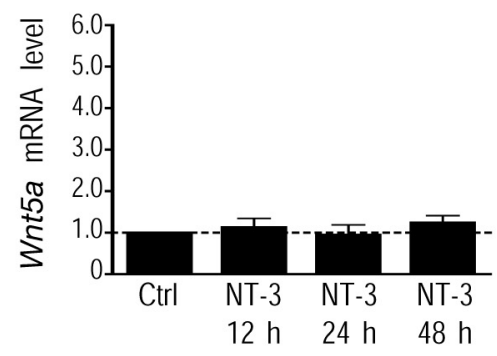

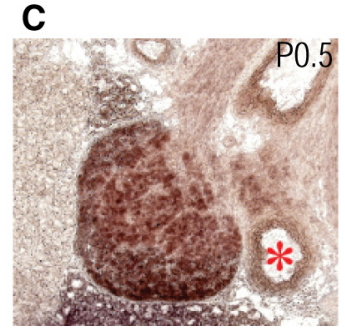

D

E

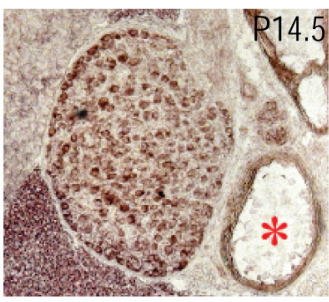

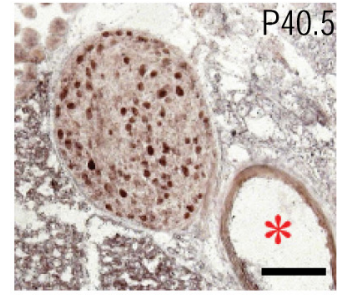

H

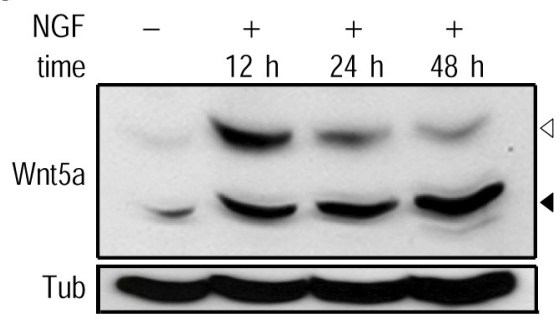

I

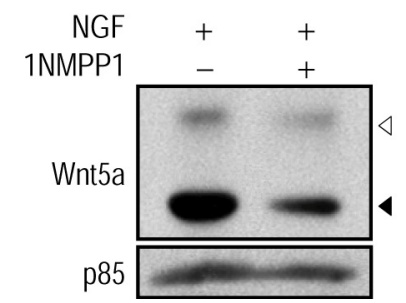

Figure 1. NGF:TrkA signaling regulates Wnt5a expression in developing sympathetic neurons. A-E, In situ hybridization analyses show expression of Wnt5a in the developing SCG. The SCG is outlined in dashed lines in $A$; the asterisk (*) identifies a neighboring blood vessel. Scale bar, $200 \mu \mathrm{m}$. $F, G$, Real-time PCR analyses show an increase in Wnt5a transcript levels in sympathetic neurons cultured for 12, 24, and $48 \mathrm{~h}$, in the presence of NGF $(100 \mathrm{ng} / \mathrm{ml})(\boldsymbol{F})$, but not NT-3 $(100 \mathrm{ng} / \mathrm{ml})(\boldsymbol{G})$. For each time point, Wnt5a levels in neurotrophin-treated cultures were compared with untreated control cultures grown in the absence of growth factor and kept viable with BAF. No changes in Wnt5a levels were observed in control cultures over the course of the experiment. Values are the mean \pm SEM from six independent experiments. For NGF-treated cultures, $12 \mathrm{~h},{ }^{* *} p=0.0068 ; 24 \mathrm{~h},{ }^{* *} p=0.0075 ; 48 \mathrm{~h},{ }^{*} p=0.0412 ;$ for NT-3-treated cultures, $12 \mathrm{~h}, p=0.5657 ; 24 \mathrm{~h}, p=$ $0.8411 ; 48 \mathrm{~h}, p=0.2085$, as determined by a two-tailed Student's t test. $\boldsymbol{H}$, NGF treatment increases Wnt5a protein levels in cultured sympathetic neurons as determined by immunoblotting. Wnt5a immunoblots were stripped and reprobed with anti- $\alpha$-tubulin as a loading control. II, Inhibition of TrkA kinase activity with the small molecule inhibitor $1 \mathrm{NMPP} 1$ (100 nm; $12 \mathrm{~h}$ ) reduces Wnt5a protein levels in TrkA ${ }^{\mathrm{F} 592 \mathrm{~A}}$ sympathetic neurons grown in the presence of NGF. The Wnt5a blot was stripped and reprobed for the p85 subunit of PI3-K (phosphatidylinositol 3-kinase) for normalization. The Wnt5a antibody recognizes a doublet of $\sim 45 \mathrm{kDa}$ (white arrowheads) and $40 \mathrm{kDa}$ (black arrowheads).

\section{Wnt5a promotes axon branching in developing sympathetic neurons}

Given that NGF regulates Wnt5a expression, we asked whether Wnt5a treatment of sympathetic neurons would elicit effects similar to those ascribed to NGF such as axon growth and survival. To test a possible role for Wnt5a in sympathetic axonal growth and morphology, we grew low-density cultures of dissociated sympathetic neurons in conditioned medium obtained from Wnt5a-expressing fibroblasts (supplemental Fig. 4, available at www.jneurosci.org as supplemental material) in the presence or absence of NGF. As controls, cultures were grown in conditioned media obtained from the parental fibroblasts used to generate the Wnt5a-expressing cell line. Cells grown in the absence of NGF were treated with BAF to prevent apoptosis. Neuronal morphology was assessed by $\beta$-III-tubulin immunostaining 8 and $24 \mathrm{~h}$ after plating cells. At $8 \mathrm{~h}$, Wnt5a elicited substantial axonal branching (Fig. $2 \mathrm{~B}$ ). Control medium-treated neurons averaged $4.29 \pm 0.517$ branches per neuron (mean \pm SEM). In the presence of Wnt5a, axon branching increased almost threefold to $12.708 \pm 0.755$ (Fig. $2 E)(p<0.001$, one-way ANOVA with Tukey-Kramer's post hoc test). Neurite length was comparable between control and Wnt5a-treated neurons, $160.8 \pm 7.4$ and $188 \pm 6.11 \mu \mathrm{m}$, respectively (Fig. $2 F)(p>0.05$, one-way
ANOVA with Tukey-Kramer's post hoc test). In contrast to Wnt5a, treatment with NGF for 8 h (Fig. 2C) had no significant effect on axon branching (Fig. $2 E$ ) but caused a substantial increase in neurite length (Fig. $2 F)(p<0.05$, one-way ANOVA with Tukey-Kramer's post hoc test). Over a 24 h period, NGFtreated neurons (Fig. 2I) exhibited the elaborate branching morphologies seen in Wnt5a-treated neurons (Fig. $2 \mathrm{H}$ ) with $23.002 \pm 2.507$ and $22.616 \pm 1.031$ branch points per neuron, respectively (Fig. $2 \mathrm{~K}$ ), although NGF-treated neurons were significantly longer than control- and Wnt5a-treated neurons (Fig. $2 L$ ) (control neurons, $263 \pm 21.7 \mu \mathrm{m}$; Wnt5a-treated neurons, $316 \pm 10.6 \mu \mathrm{m}$; NGF-treated neurons, $380 \pm 17.2 \mu \mathrm{m} ; p<0.05$, one-way ANOVA with Tukey-Kramer's post hoc test). Thus, whereas the effects of Wnt5a on axonal branching are observed within $8 \mathrm{~h}$, NGF-induced axonal arborization is observed only after $24 \mathrm{~h}$. The effect of NGF on axonal branching is similar to, but not additive with, Wnt5a, since addition of NGF to Wnt5atreated neurons did not further augment axonal arborization (Figs. $2 E, K$ ). At $24 \mathrm{~h}$, there were no significant differences in neurite length between control and Wnt5a-treated neurons (Fig. $2 L$ ) indicating a specific effect of Wnt5a on axonal branching.

To test a possible role for Wnt5a in neuronal survival, we grew low-density cultures of dissociated sympathetic neurons in 


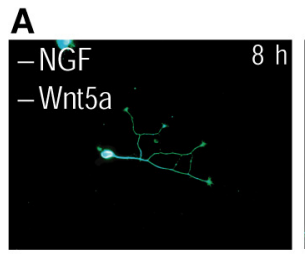

B
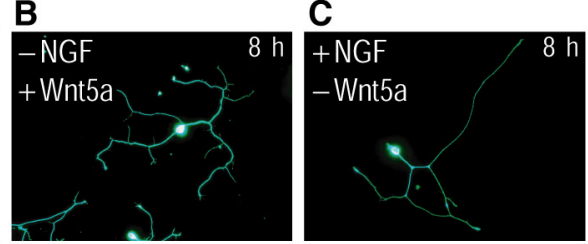

D
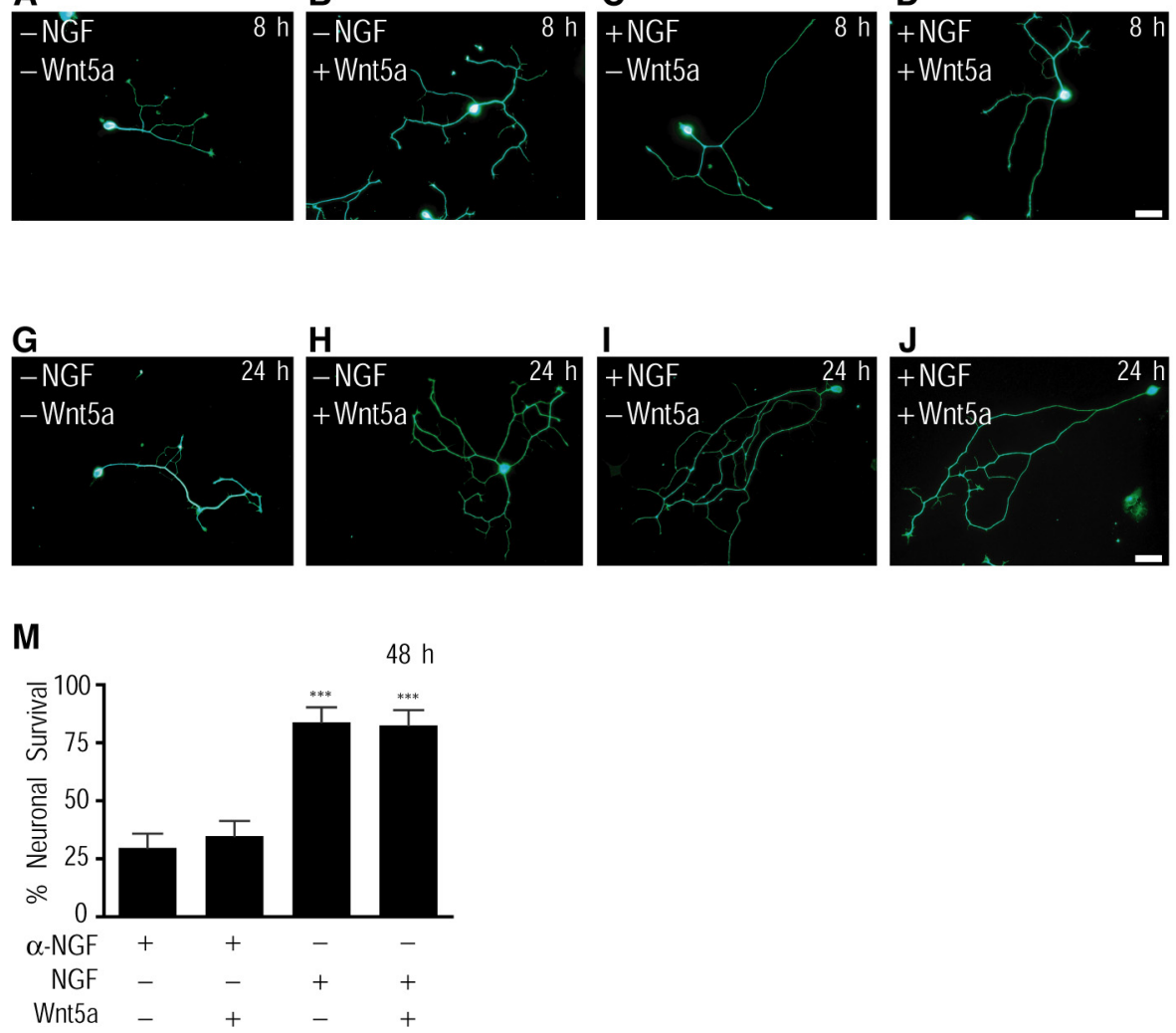
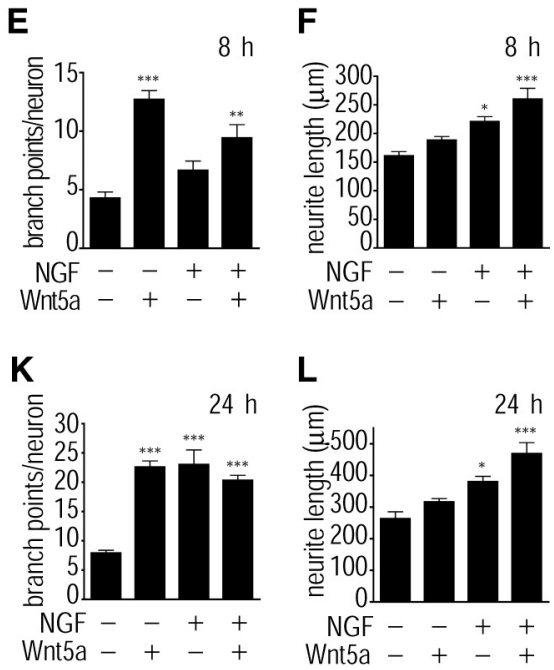

Figure 2. Wnt5a promotes axonal branching and growth. $\boldsymbol{A}-\boldsymbol{D}, \beta$-III-Tubulin staining shows morphologies of sympathetic neurons cultured for $8 \mathrm{~h}$ in control media $(\boldsymbol{A})$, Wnt5a $(\boldsymbol{B}), \mathrm{NGF}(\boldsymbol{C})$, or NGF plus Wnt5a (D)-containing media. Scale bar, $50 \mu \mathrm{m}$. $\boldsymbol{E}, \boldsymbol{F}$, Quantification of branch points per neuron $(\boldsymbol{E})$ and neurite length $(\boldsymbol{F})$ in low-density dissociated cultures treated as described in $\boldsymbol{A}-\boldsymbol{D}$. Values are the mean \pm SEM from five independent experiments. ${ }^{*} p<0.05$, ${ }^{* *} p<0.01$, and ${ }^{* * *} p<0.001$, significantly different from control media-treated neurons, as determined by one-way ANOVA followed by Tukey's multiple-comparisons test. $G-J$, $\beta$-III-Tubulin staining shows morphologies of sympathetic neurons cultured for $24 \mathrm{~h}$ in control media (G), Wnt5a (H), NGF (I), or NGF plus Wnt5a $(\boldsymbol{J})$-containing media. Scale bar, $50 \mu \mathrm{m} . \boldsymbol{K}, \boldsymbol{L}$, Quantification of branch points per neuron $(\boldsymbol{K})$ and neurite length $(\boldsymbol{L})$ in low-density dissociated cultures treated as described in $\mathbf{G}-\boldsymbol{J}$. Values are the mean \pm SEM from five independent experiments. ${ }^{*} p<0.05$ and ${ }^{* * *} p<0.001$, significantly different from control medium-treated neurons, as determined by one-way ANOVA followed by Tukey's multiple-comparisons test. M, Quantification of percentage neuronal survival in dissociated cultures treated with anti-NGF, anti-NGF plus Wnt5a, NGF, or NGF plus Wnt5a for $48 \mathrm{~h}$. Neurons were initially grown for $4-5 \mathrm{~d}$ in NGF (10 ng/ml)-containing media and then shifted to the different conditions described above. Neuronal survival was determined by Hoechst staining to visualize nuclear morphology. Values are the mean \pm SEM from four independent experiments. ${ }^{* *} p<0.001$, significantly different from anti-NGF-treated neurons, as determined by one-way ANOVA followed by Tukey's multiple-comparisons test.

Wnt5a or control conditioned medium in the presence or absence of NGF for 48 h. Percentage neuronal survival was determined by Hoechst staining to visualize nuclear morphology. Wnt5a treatment did not promote neuronal survival, with only $34 \%$ of neurons surviving, comparable with the $29 \%$ survival observed when neurons were cultured in the absence of NGF. In contrast, treatment of neurons with NGF (10 ng/ml), in the presence or absence of Wnt5a, supported neuronal survival at $\sim 85 \%$ (Fig. $2 M$ ). These results indicate that Wnt5a is not sufficient to promote sympathetic neuron survival in the absence of NGF.

\section{Wnt5a-mediated axon branching is rapid and transcription independent}

To visualize how sympathetic axons develop in response to Wnt5a, we performed time-lapse imaging of Wnt5a-treated neurons at $30 \mathrm{~min}$ intervals for up to $2 \mathrm{~h}$. Neurons were initially plated and cultured in control conditioned medium for $8 \mathrm{~h}$, and then stimulated with Wnt5a media. Wnt5a had immediate and pronounced effects on axon branching. As early as $30 \mathrm{~min}$ after Wnt5a addition, neurons exhibited distinct sprouting and extension of branches (Fig. $3 G, K$ ), and after $2 \mathrm{~h}$ of imaging, most Wnt5a-treated neurons had elaborate multibranched arbors (Fig. 3J). The number of newly formed branches increased with time such that Wnt5a-treated neurons ( $n=16$ neurons) aver- aged $18.64 \pm 2.1$ branches per neuron after $2 \mathrm{~h}$ of imaging, an increase of $188 \%$ (Fig. $3 F-K$ ). In neurons treated with control conditioned medium ( $n=18$ neurons), the rate and extent of branching was slow, averaging $9.88 \pm 0.55$ branches per neuron after $2 \mathrm{~h}$ (Fig. $3 A-E, K)$. Although the newly formed branches in Wnt5a-treated neurons showed rapid extension, the primary neurites grew to a similar extent as in control medium-treated neurons, such that we observed no obvious differences in length of the longest neurite between the two groups after $2 \mathrm{~h}$.

These rapid effects indicate that Wnt5a might induce axon remodeling by regulating cytoskeletal effectors, independent of transcription. To directly test the role of transcription in the action of Wnt5a, neurons were first cultured in control conditioned medium for $8 \mathrm{~h}$ to allow neurite outgrowth and then exposed to Wnt5a in the presence of the transcription inhibitor actinomycin $\mathrm{D}(0.1 \mu \mathrm{g} / \mathrm{ml})$ for $16 \mathrm{~h}$. This concentration of actinomycin D has been shown previously to completely block transcription in sympathetic neuron cultures (Franklin and Johnson, 1998). Actinomycin D had no effect on Wnt5a-induced axon branching (Fig. $3 L$ ), indicating a transcription-independent mode of action for Wnt5a. To test the translational requirements for the rapid effects of Wnt5a on axon branching, we initially cultured neurons overnight in control conditioned medium to allow formation of neurites. Subsequently, we treated cultures with control or Wnt5a- 
A

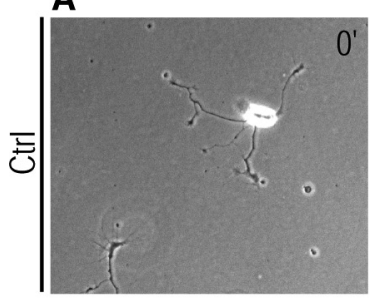

F

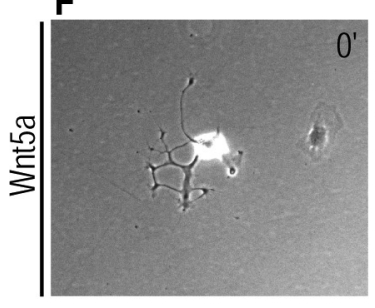

K

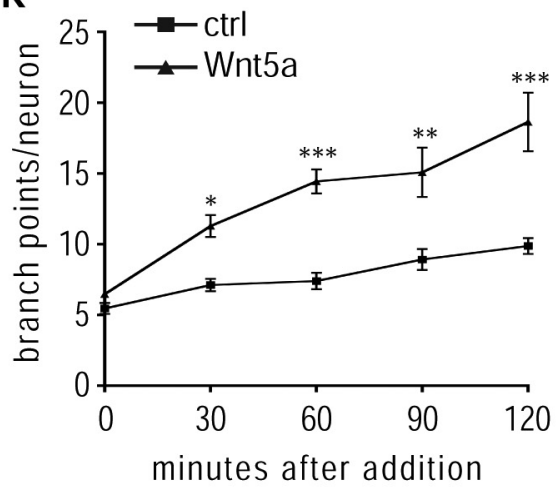

C

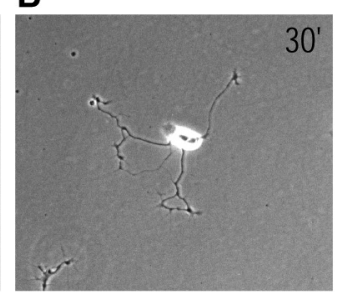

G

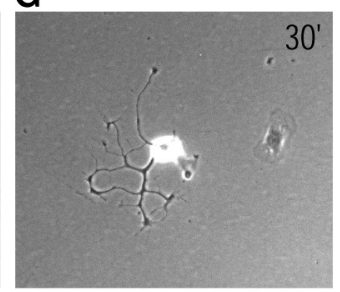

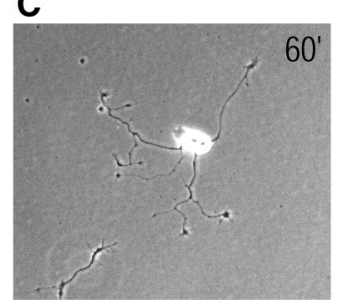

H

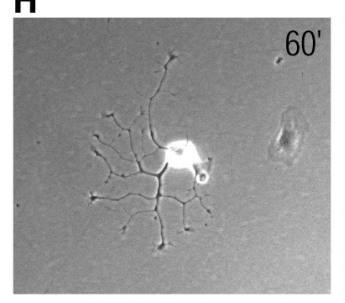

D

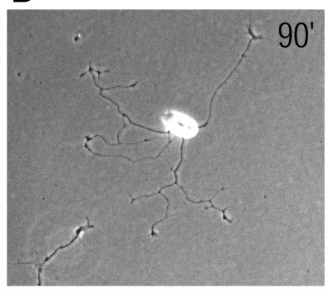

I

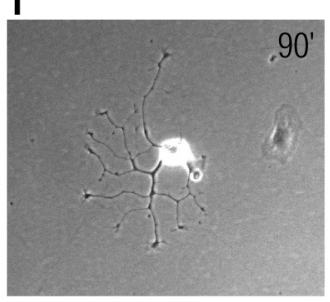

E

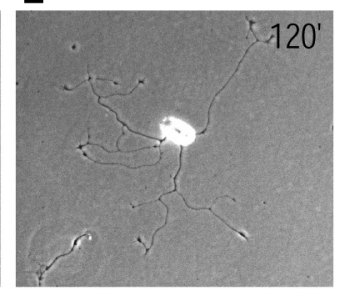

J

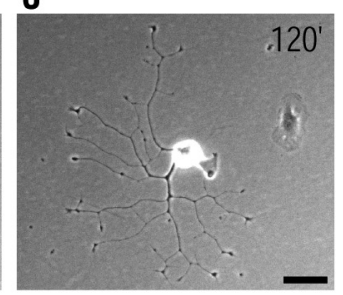

L

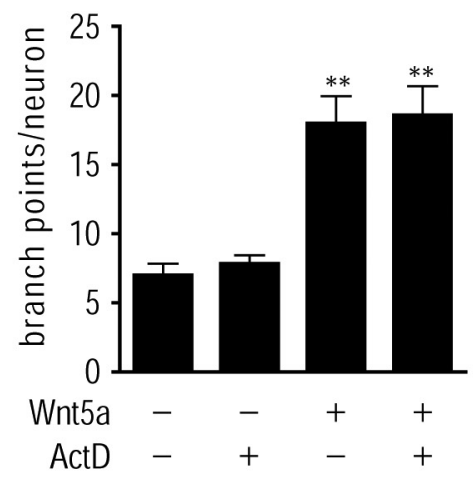

M

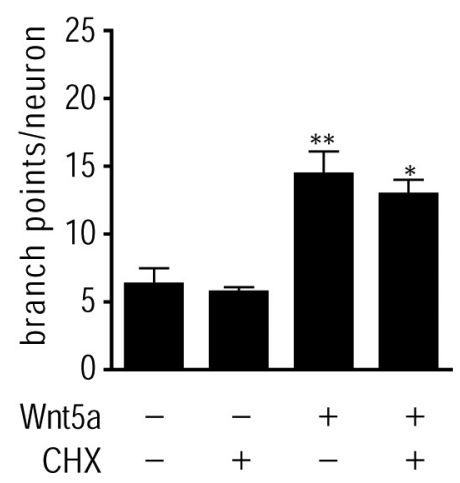

Figure 3. Wnt5a elicits rapid effects on axon branching, independent of transcription and translation. $A-E$, Phase-contrast images of a sympathetic neuron treated with control media for 30 min $(\boldsymbol{B}), 60 \mathrm{~min}(\boldsymbol{C}), 90 \mathrm{~min}(\boldsymbol{D})$, and $120 \mathrm{~min}(\boldsymbol{E}) . \boldsymbol{F}-J$, Phase-contrast images of a sympathetic neuron treated with Wnt5a-containing media for $30 \mathrm{~min}(\boldsymbol{G}), 60 \mathrm{~min}(\boldsymbol{H}), 90 \mathrm{~min}(\boldsymbol{I})$, and $120 \mathrm{~min}(\boldsymbol{J})$. Neurons were initially plated in control conditioned medium for $8 \mathrm{~h}$, and then treated with Wnt5a or control media for up to $2 \mathrm{~h}$. Scale bar, $40 \mu \mathrm{m}$. $K$, Quantification of axonal branch points in neurons treated as described in $A-J$. Results are the mean \pm SEM from a total of three independent experiments with six to eight neurons imaged per condition for each experiment. ${ }^{*} p<0.05$, ${ }^{* *} p<0.01$, ${ }^{* * *} p<0.001$, significantly different from control medium-treated neurons, as determined by one-way ANOVA followed by Tukey's multiple-comparisons test. $L$, Quantification of axonal branch points in neurons treated with control or Wnt5a media in the presence or absence of actinomycin D $(0.1 \mu \mathrm{g} / \mathrm{ml})$. Neurons were initially plated in control conditioned medium for $8 \mathrm{~h}$ and then treated with Wnt5a or control media in the presence or absence of actinomycin $D(0.1 \mu \mathrm{g} / \mathrm{ml})$ for $16 \mathrm{~h}$. Neurons were then fixed and immunostained with $\beta$-III-tubulin. ${ }^{* *} p<0.01$, significantly different from control medium-treated neurons, as determined by one-way ANOVA followed by Tukey's multiple-comparisons test. $M$, Quantification of axonal branch points in neurons treated with control or Wnt5a media in the presence or absence of cycloheximide $(1 \mu \mathrm{g} / \mathrm{ml})$. Neurons were initially plated in control conditioned medium overnight to allow extension of neurites, and then treated for $1 \mathrm{~h}$ with Wnt5a or control media in the presence or absence of cycloheximide $(1 \mu \mathrm{g} / \mathrm{ml}$; pretreatment, $15 \mathrm{~min})$. Neurons were then fixed and immunostained with $\beta$-III-tubulin. ${ }^{*} p<0.05$ and ${ }^{* *} p<0.01$, significantly different from control medium-treated neurons, as determined by one-way ANOVA followed by Tukey's multiple-comparisons test.

containing media for $1 \mathrm{~h}$ in the presence or absence of the translational inhibitor cycloheximide $(1 \mu \mathrm{g} / \mathrm{ml}$; pretreatment, 15 min). Cycloheximide treatment did not affect the ability of Wnt5a to induce short-term axon branching (Fig. 3M), suggesting a translation-independent mechanism potentially via local regulation of cytoplasmic effectors. Together, these experiments demonstrate a potent, rapid, and transcription/translationindependent effect of Wnt5a on the rate and extent of axon branching in developing sympathetic neurons.

\section{Localized effects of Wnt5a on axon branching and growth}

To determine whether Wnt5a could locally induce changes in axon morphology and growth, Wnt5a was added directly to axon terminals of compartmentalized cultures of sympathetic neurons. In this culture system, neuronal cell bodies and axon terminals are segregated into distinct fluid compartments by a Teflongrease barrier, allowing addition of growth factors directly to axons (Campenot, 1977, 1979). Wnt5a-treated axons showed strikingly elaborate morphologies with significantly enhanced arborization (Fig. $4 B$ ), whereas hardly any branching was seen in sympathetic axons treated with control media (Fig. $4 A$ ). Wnt5atreated neurons had on average $16.38 \pm 2.99$ branch points per axon compared with $6.69 \pm 1.72$ branch points per axon for control neurons ( $p=0.0267$, unpaired, two-tailed Student's $t$ test). The complex pattern of arborization seen in Wnt5a-treated axons in vitro is reminiscent of the morphological changes that sympathetic axons undergo in vivo on innervation of final target tissues (Glebova and Ginty, 2004). Wnt5a added exclusively to axon compartments also significantly enhanced axonal extension (in micrometers per day) into side compartments of compartmentalized cultures over the $72 \mathrm{~h}$ period of monitoring axon growth (Wnt5a, $291.05 \pm 32.96 \mu \mathrm{m} / \mathrm{d}$, vs control, $172.32 \pm 10.78$ $\mu \mathrm{m} / \mathrm{d} ; p=0.0485$, unpaired, two-tailed Student's $t$ test). Thus, whereas the effects of Wnt5a on axonal branching are observed within $8 \mathrm{~h}$, Wnt5a-induced increases in axon elongation are observed after $3 \mathrm{~d}$ of exposure to the Wnt ligand. 


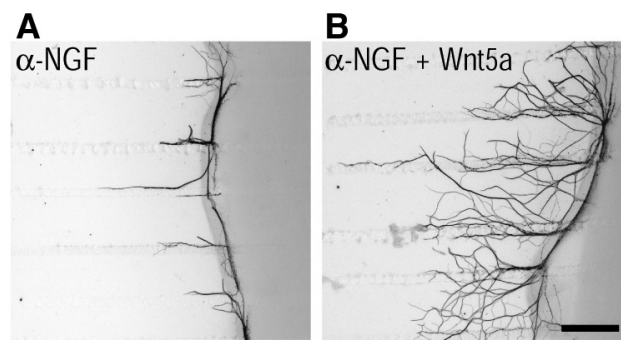

C
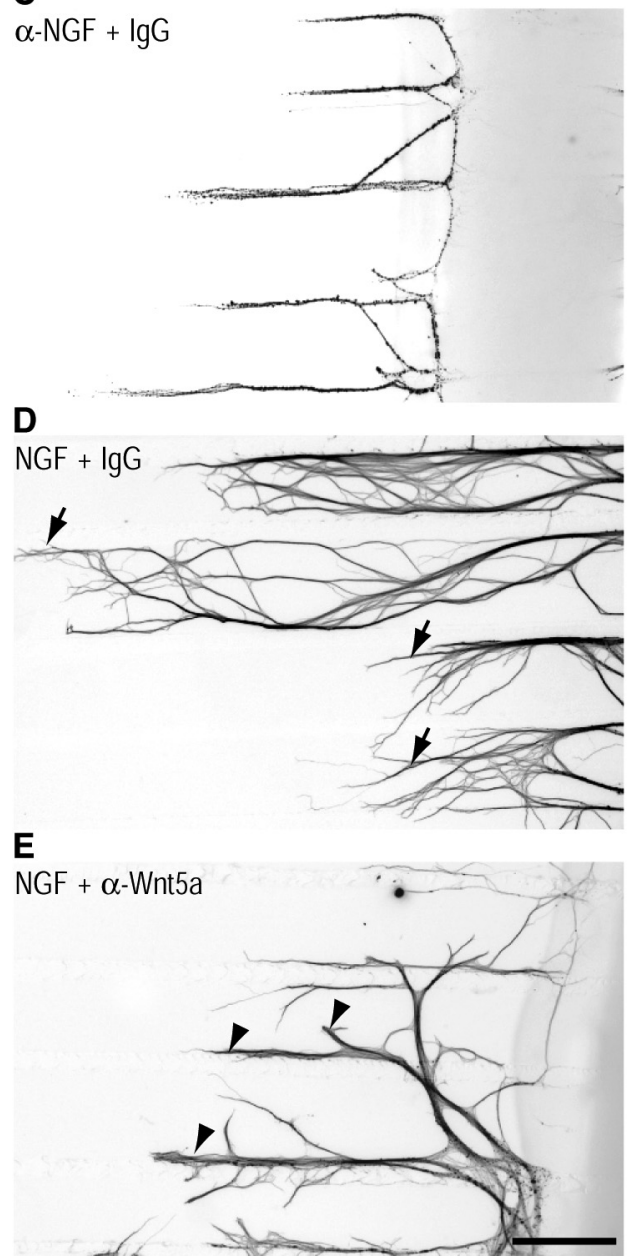

$\mathbf{F}$

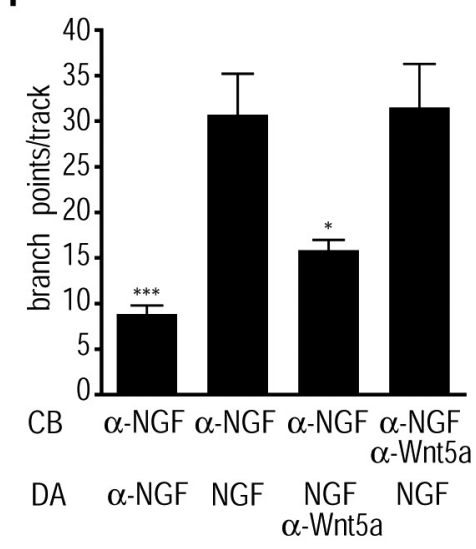

G

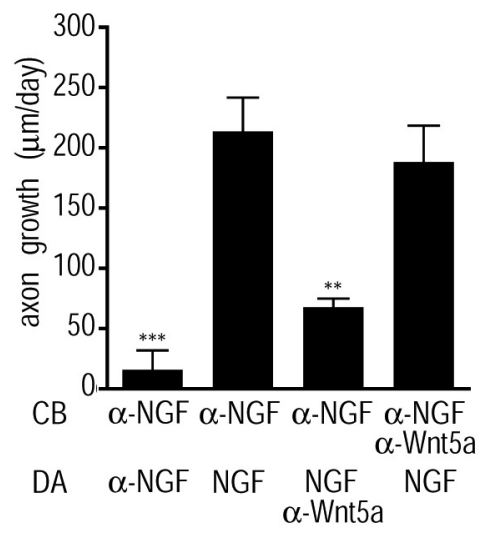

Figure 4. Localized effects of Wnt5a on axon branching and growth. $\boldsymbol{A}, \boldsymbol{B}$, Wnt5a added directly to axons promotes axonal branching and axonal extension over $3 \mathrm{~d}$. In compartmentalized cultures, Wnt5a-treated axons $(\boldsymbol{B})$ exhibit robust axonal arborization and growth, compared with axons treated with control medium $(\boldsymbol{A})$. Images of $\beta$-III-tubulin immunostaining are inverted. Scale bar, $330 \mu \mathrm{m}$. C-E, Neutralizing Wnt5a in axon compartments attenuates NGF-dependent growth. NGF added exclusively to sympathetic axons in compartmentalized cultures elicits axonal extension and branching responses ( $\boldsymbol{D}$, arrows) that are eliminated by the inclusion of a neutralizing antibody to NGF (C). Sympathetic axons treated with NGF in the presence of a Wnt5a neutralizing antibody were shorter and highly fasciculated, with little branching at their terminals (E, arrowheads). Scale bar, 330 $\mu \mathrm{m} . \boldsymbol{F}, \mathbf{G}$, Quantification of branch points per track $(\boldsymbol{F})$ and axonal growth (in micrometers per day) ( $\boldsymbol{G}$ ) in low-density compartmentalized cultures treated as indicated in $\boldsymbol{C}-\boldsymbol{E}$ except in the last condition in which neutralizing Wnt5a antibody was added to the cell body (CB) compartments. Values are the mean \pm SEM from six independent experiments. ${ }^{*} p<0.05,{ }^{* *} p<0.01$, and ${ }^{* * *} p<0.001$, as determined by one-way ANOVA followed by Tukey's multiple-comparisons test.

If NGF regulates Wnt5a expression in sympathetic neurons to mediate axonal growth and branching, then depleting endogenous Wnt5a should interfere with the growth-promoting abilities of NGF. To block endogenous Wnt5a signaling, we used an antibody that has been previously shown to neutralize Wnt5a activity in hematopoietic stem cells (Murdoch et al., 2003). In compartmentalized cultures, NGF added exclusively to axon terminals of sympathetic axons elicits axonal extension and branching responses (Fig. 4D). Consistent with previous studies, the axonal effects of NGF were completely eliminated by the inclusion of a neutralizing antibody to NGF (Fig. 4C) (Kuruvilla et al., 2004). Sympathetic axons treated with NGF in the presence of the Wnt5a neutralizing antibody were shorter and highly fasciculated, with little branching at their terminals (Fig. 4E). These results suggest that the Wnt5a synthesized by sympathetic neurons is secreted and that it acts downstream of NGF to mediate axonal extension and branching. The neutralizing Wnt5a antibody antagonized NGFdependent axonal branching (Fig. $4 F$ ) and growth (Fig. 4G) only when added to distal axons and had no significant effect when added directly to cell bodies. Together with our findings that Wnt5a promotes branching within minutes and that it acts via a transcription-independent mechanism (Fig. $3 A-L$ ), these results point to a local effect of Wnt5a in distal axons.

\section{NGF-dependent axonal branching and growth is reduced in sympathetic neurons lacking Wnt5a}

To directly test the role of Wnt5a as a downstream effector of NGF-dependent axonal branching, we next analyzed the ability of neurons lacking Wnt5a to grow in response to NGF. NGF-dependent axonal growth was assessed in SCG explants and dissociated sympathetic neurons isolated from $W n t 5 a^{-1-}$ and wild-type mice. SCG explants $(n=8$ explants for each genotype) harvested from E18.5 wild-type and $W n t 5 a^{-1-}$ mice were grown for $72 \mathrm{~h}$ in collagen gel in the presence of NGF. Whereas control explants exhibited robust NGF-dependent axonal growth as evident from a broad axonal halo (Fig. 5A), $W n t 5 a^{-1-}$ explants showed poor outgrowth (Fig. 5B), despite the presence of NGF in the culture media. Neurite outgrowth, as quantified by measuring the area covered by the axons of each explant relative to the area occupied by the cell bodies, indicated a significant 1.6-fold reduction in growth by $W n t 5 a^{-1-}$ explants ( $p=0.029$, unpaired, two-tailed Student's $t$ test).

Low-density cultures of dissociated sympathetic neurons were established from $W n t 5 a^{-l-}$ and wild-type mice, and their morphology examined after $24 \mathrm{~h}$ in culture by immunostaining for $\beta$-IIItubulin. For the majority of wild-type neurons, a single axonal shaft emerged from a neuronal cell body, which then ramified into secondary branches and finer collaterals (Fig. 5C). In wildtype neurons, the main axonal shaft and secondary branches had several filopodia-like extensions that were longer than $5 \mu \mathrm{m}$. In 
A

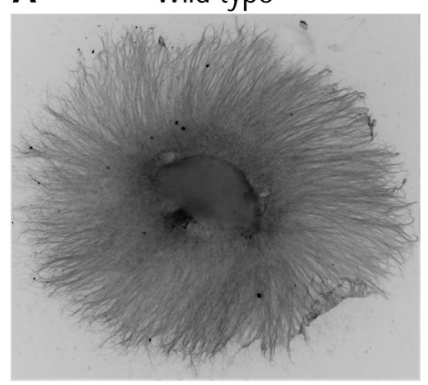

B
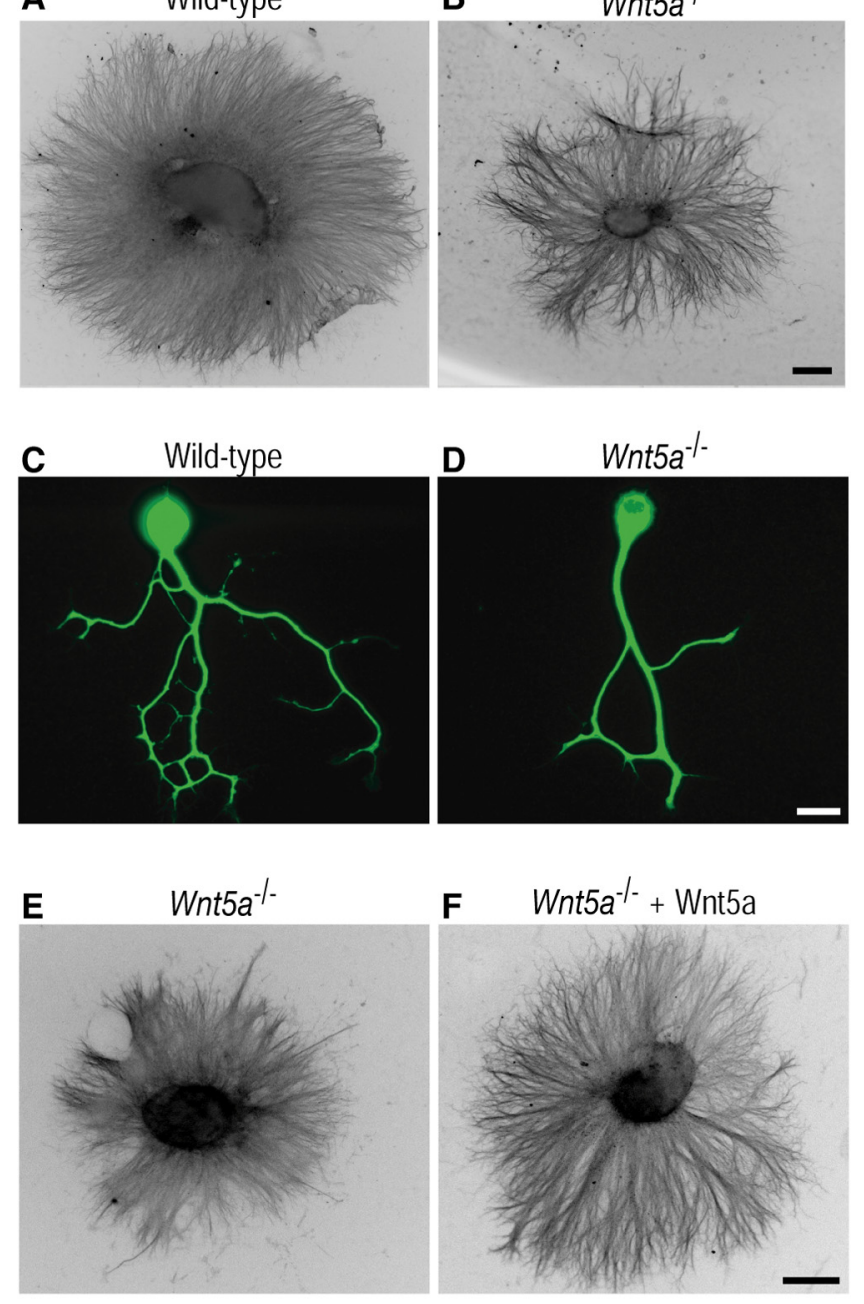

G
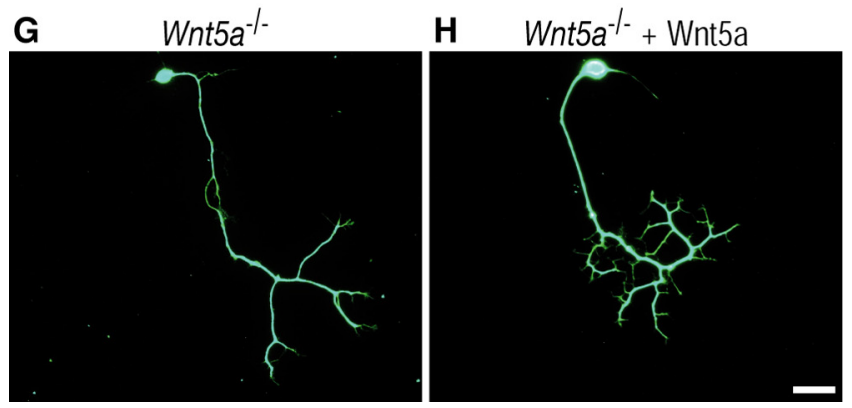

Figure 5. Sympathetic neurons lacking Wnt5a show deficits in NGF-dependent axonal branching and growth. $\boldsymbol{A}, \boldsymbol{B}$, Reduced NGF-dependent axonal growth in SCG explants from Wnt5a $a^{-1-}$ mice. SCG explants from E18.5 wild-type $(\boldsymbol{A})$ and $W n t 5 a^{-1-}(\boldsymbol{B})$ mice were grown for 3 d in collagen gel in NGF-containing media; ganglia were then fixed and immunostained for $\beta$-IIl-tubulin. Scale bar, $320 \mu$ m. C, D, Wnt5a $a^{-/-}$sympathetic neurons show reduced branching in response to NGF. Dissociated sympathetic neurons isolated from E18.5 wild-type (C) or Wnt5a $a^{-1-}(\boldsymbol{D})$ embryos were cultured for $24 \mathrm{~h}$ in NGF-containing media and then immunostained for $\beta$-III-tubulin. Scale bar, $20 \mu \mathrm{m}$. $\boldsymbol{E}, \boldsymbol{F}$, Exogenous Wnt5a rescues the axonal growth deficits in Wnt5a $a^{-1-}$ SCG explants. SCG explants from E16.5 Wnt5a $a^{-1-}$ mice were grown for $3 \mathrm{~d}$ in collagen gel in control $(\boldsymbol{E})$ or Wnt5a $(\boldsymbol{F})$ conditioned media supplemented with NGF (50 $\mathrm{ng} / \mathrm{ml}$ ), and then immunostained for $\beta$-III-tubulin. Scale bar, $320 \mu \mathrm{m} . \boldsymbol{G}, \boldsymbol{H}$, Exogenous Wnt5a rescues the branching deficits in dissociated $W n t 5 a^{-1-}$ neurons. Dissociated sympathetic neurons isolated from E18.5 Wnt5a $\mathrm{a}^{-1-}$ mice were cultured for $24 \mathrm{~h}$ in control $(\boldsymbol{G})$ or Wnt5a $(\boldsymbol{H})$ conditioned medium supplemented with NGF $(50 \mathrm{ng} / \mathrm{ml})$, and then immunostained for $\beta$-IIItubulin. Scale bar, $40 \mu \mathrm{m}$. contrast, Wnt5a $a^{-/-}$neurons had very smooth axons with little branching along the main axonal shaft and secondary branches (Fig. 5D). Quantification of axonal branch points indicated a 2.3 -fold reduction in axonal branching in $W n t 5 a^{-1-}$ neurons ( $n=7$ independent experiments, total of 265 neurons) compared with wild-type neurons (296 neurons) ( $p=0.0004$, unpaired, two-tailed Student's $t$ test). No significant differences were observed in neurite length between wild-type and mutant neurons after $24 \mathrm{~h}$ in culture ( $p=0.0636$, unpaired, two-tailed Student's $t$ test), indicating that axon outgrowth is less sensitive to the loss of Wnt5a than axonal branching.

We reasoned that, if the loss of Wnt5a is primarily responsible for reduced axonal branching observed in $W n t 5 a^{-1-}$ neurons cultured in the presence of NGF, addition of exogenous Wnt5a should rescue the deficits. Indeed, $W n t 5 a^{-1-}$ SCG explants exposed to exogenous Wnt5a for $72 \mathrm{~h}$ showed a significant 2.3 -fold increase in axonal outgrowth compared with those treated with control medium (Fig. $5 E, F)(n=3$ explants for each condition; $p=0.027$, unpaired, two-tailed Student's $t$ test). Exogenous Wnt5a also elicited elaborate axonal arborization in dissociated $W n t 5 a^{-l-}$ sympathetic neurons, compared with those treated with control medium (Fig. 5G,H). Quantification revealed a significant increase (threefold) in axonal branching $(p=0.016$, unpaired, two-tailed Student's $t$ test), but not neurite length ( $p=$ 0.22 , unpaired, two-tailed Student's $t$ test) in $W n t 5 a^{-1-}$ neurons treated with exogenous Wnt5a in the $24 \mathrm{~h}$ culture period. These results indicate that the signaling machinery required to respond to Wnt5a-dependent branching remains intact in $W n t 5 a^{-1-}$ neurons and that the branching deficits are caused primarily by the absence of Wnt5a in these neurons.

\section{$W n t 5 a^{-1-}$ mice have deficits in the developing sympathetic nervous system}

Given the axonal branching and growth deficits observed in vitro in sympathetic neurons lacking Wnt5a, we investigated whether Wnt5a is required for SCG development and sympathetic innervation of target tissues in vivo. In $W n t 5 a^{-1-}$ mice, the SCG at E13.5 had coalesced into discrete ganglia and expressed tyrosine hydroxylase, a marker of neuronal differentiation (Fig. 6A,B) and $\beta$-III-tubulin (data not shown) at normal levels. Furthermore, from E13.5 to E15.5, a time period of robust cellular proliferation (Fagan et al., 1996), the Wnt5a ${ }^{-1-}$ SCG has a similar number of cells compared with wild type, suggesting early stages of differentiation and proliferation are unperturbed by $\mathrm{Wnt5a}$ loss (Fig. 6A-D,I). However, at E17.5, when sympathetic neuronal survival is dependent on NGF, we observed a substantial (34\%) decrease in $W n t 5 a^{-1-}$ SCG cell number that progressed throughout P0.5 (Fig. $6 E-I)(p<0.01$ for E17.5 and $p<0.05$ for P0.5, unpaired two-tailed Student's $t$ test), similar to the timing and magnitude of developmental cell loss documented in $N G F^{-1-}$ (Brennan et al., 1999; Francis et al., 1999) and TrkA ${ }^{-1-}$ mice (Fagan et al., 1996). For comparison, in both $N G F^{-1-}$ and $\operatorname{Trk} A^{-1-}$ mice, a significant reduction in neuronal number is first detected at E17.5, with a $39-61 \%$ decrease in the $N G F^{-1-}$ mice (Brennan et al., 1999; Francis et al., 1999) and 35\% decrease in TrkA $A^{-1-}$ mice (Fagan et al., 1996) compared with wild-type mice.

Given the decrease in SCG cell number at E17.5, we performed immunocytochemistry against tyrosine hydroxylase and an apoptotic marker, cleaved caspase-3, in the wild-type and $W n t 5 a^{-1-}$ SCG. Quantification of the percentage of caspase-3-positive cells in E15.5 wild-type $(6.8 \pm 1.6 \%)$ and $W n t 5 a^{-1-}(7.4 \pm 2.5 \%)$ SCG indicated there is no significant difference in levels of apo- 

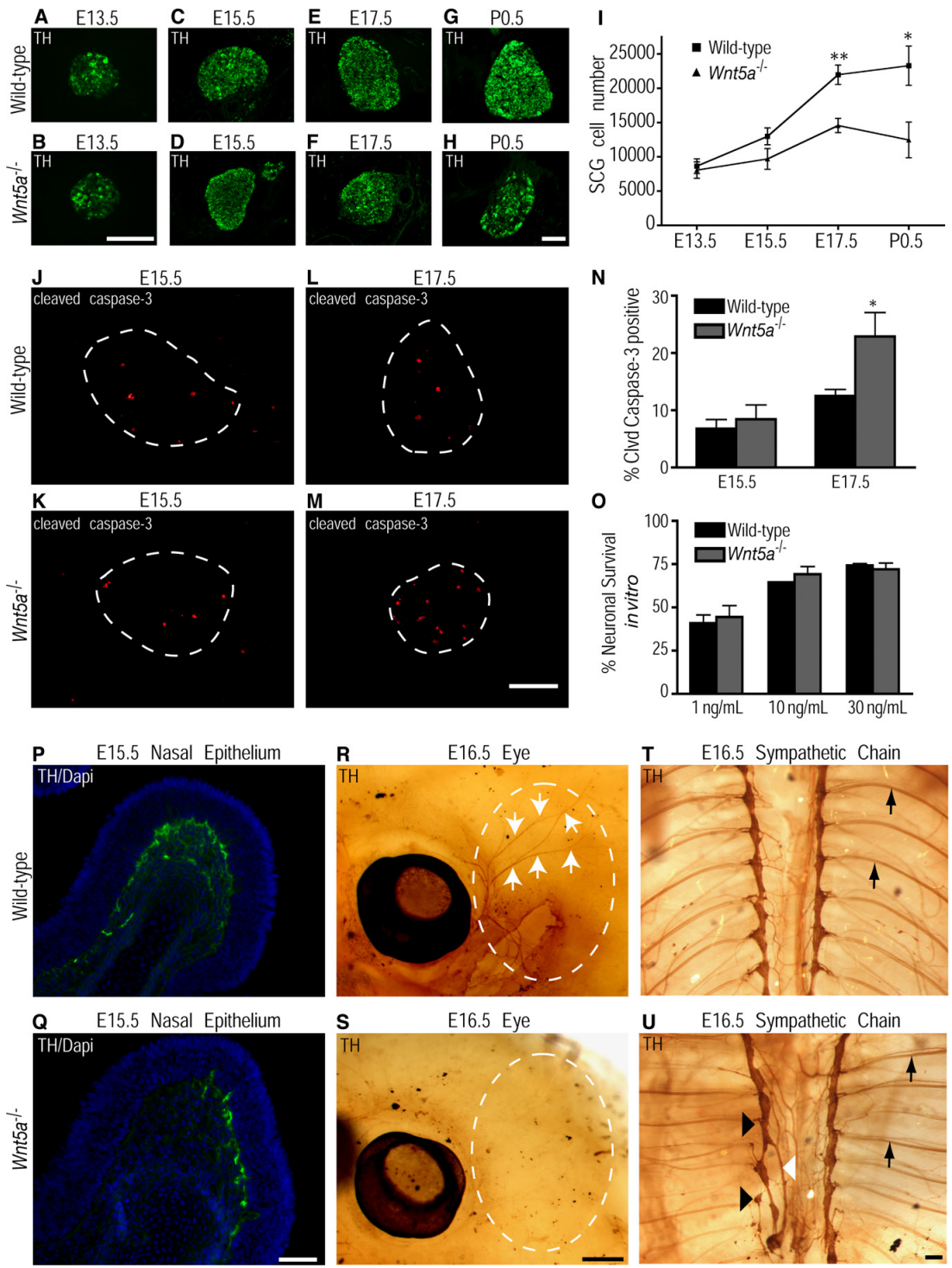

$\mathbf{N}$

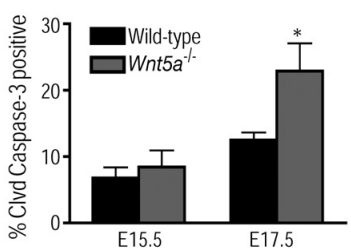

0
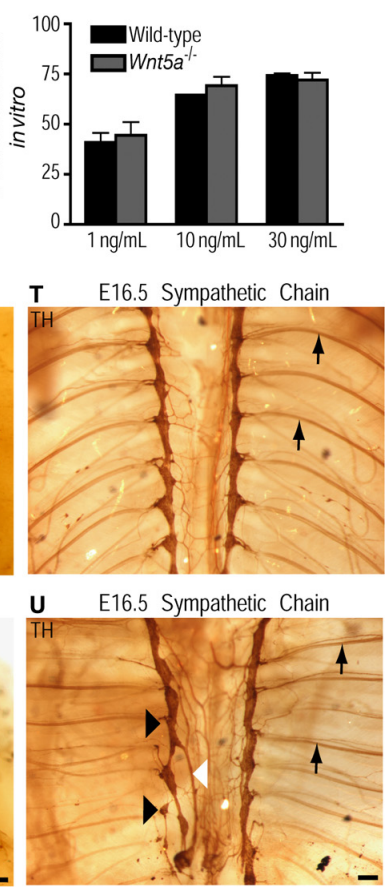

Figure 6. Wnt5a $a^{-1-}$ mice show reduced innervation of SCG targets, enhanced apoptosis in the SCG, and abnormalities in sympathetic chain formation. $\boldsymbol{A}-\boldsymbol{H}$, Tyrosine hydroxylase immunocytochemistry of wild-type and $W n t 5 a^{-1-}$ SCG at E13.5 $(\boldsymbol{A}, \boldsymbol{B})$, E15.5 (C, D), E17.5 $(\boldsymbol{E}, \boldsymbol{F})$, and P0.5 $(\boldsymbol{G}, \boldsymbol{H})$. Scale bar, $100 \mu \mathrm{m}$. I, Quantification of SCG cell number in wild-type and Wnt5a embryos at E13.5, E15.5, E17.5, and P0.5 by Nissl staining and cell counting show that a significant reduction in cell number is first evident in the mutants at E17.5 and progresses through P0.5. Values are the mean \pm SEM ( $n=7$ embryos for E13.5, $n=5$ for E15.5, $n=4$ for E17.5, and $n=3$ for P0.5 for each genotype). ${ }^{* *} p<0.01$ (E17.5) and ${ }^{*} p<0.05$ (P0.5) as determined by unpaired, two-tailed Student's t test. J-M, Cleaved caspase-3 immunocytochemistry of wild-type and Wnt5a ${ }^{-1-}$ SCG atE15.5 (J, $\boldsymbol{K})$ and E17.5 $(\boldsymbol{L}, \boldsymbol{M})$. Scale bar, $100 \mu \mathrm{m}$. N, Quantification of the percentage apoptotic cells in the SCG of wild-type and Wnt $5 a^{-1-}$ embryos at E15.5 and E17.5 by counting the number of cleaved caspase-3-positive cells as a percentage of all tyrosine hydroxylase-positive cells. Values are the mean \pm SEM for each genotype (E15.5, $n=5 ;$ E17.5, $n=7) .{ }^{*} p=0.0175$ (E17.5) as determined by an unpaired two-tailed Student's $t$ test. $\mathbf{0}$, Quantification of percentage neuronal survival of dissociated sympathetic neurons from E15.5 wild-type and Wnt5a $a^{-1-}$ SCG cultured in the presence of 1,10 , and $30 \mathrm{ng} / \mathrm{ml}$ NGF. Values are the mean \pm SEM of four independent experiments. $\boldsymbol{P}, \mathbf{Q}$, Tyrosine hydroxylase immunocytochemistry and $4^{\prime}, 6^{\prime}$-diamidino-2phenylindole (DAPI) staining of the nasal epithelium in wild-type $(\boldsymbol{P})$ and $W n t 5 a^{-1-}(\mathbf{Q})$ E15.5 embryos reveals reduced innervation in the absence of Wnt5a (tyrosine hydroxylase is shown in green and DAPI in blue). Scale bar, $50 \mu \mathrm{m} . \boldsymbol{R}, \mathbf{S}$, Whole-mount tyrosine hydroxylase staining of the eye in wild-type $(\boldsymbol{R})$ and $W n t 5 a^{-1-}(\boldsymbol{S})$ E16.5 mice indicates that SCG neurons fail to innervate this target in Wnt5a $a^{-1-}$ embryos (white arrows). Scale bar, $500 \mu \mathrm{m}$. $\boldsymbol{T}, \boldsymbol{U}$, Whole-mount tyrosine hydroxylase immunostaining of E16.5 wild-type $(\boldsymbol{T})$ and $W n t 5 a^{-1-}(\boldsymbol{U})$ embryos show a disorganized sympathetic chain in the absence of Wnt5a (black arrowheads indicate fused or ectopic ganglia; white arrowhead indicates abnormal axonal projections; black arrows indicate proximal projections along intercostal arteries). Scale bar, $100 \mu \mathrm{m}$.

ptosis at E15.5 (Fig. $6 J, K, N$ ). In contrast, at E17.5, the percentage of cleaved caspase-3-positive cells is significantly higher in the Wnt5a ${ }^{-1-}$ SCG $(22.9 \pm 4.3 \%)$ compared with wild type (12.5 \pm $1.2 \%$ ) (Fig. $6 L-N$ ). Thus, enhanced SCG apoptosis and reduc- tion in cell number in $W n t 5 a^{-1-}$ embryos occurs at a developmental time when axons are actively innervating target tissues and gaining access to NGF.

To determine whether there is a cellautonomous requirement for Wnt5a in neuronal survival, we isolated sympathetic neurons from wild-type and Wnt $5 a^{-/-}$ SCGs at E15.5, before the onset of enhanced apoptosis seen in vivo, and maintained dissociated neurons in different concentrations of NGF $(1,10$, and $30 \mathrm{ng} /$ $\mathrm{ml}$ ) for $4 \mathrm{~d}$ in vitro. Although NGF at 10 and $30 \mathrm{ng} / \mathrm{ml}$ was more effective than 1 $\mathrm{ng} / \mathrm{ml}$ at promoting neuronal survival in both wild-type and Wnt5a $a^{-1-}$ neurons, we did not see any significant differences in sensitivity to NGF between the two genotypes. Quantification of NGFdependent neuronal survival showed equivalent numbers of surviving Wnt $5 a^{-1-}$ and wild-type neurons at each NGF concentration (Fig. 6O). These results indicate that Wnt5a is not primarily required to mediate NGF-dependent neuronal survival.

In light of the requirement for Wnt5a in NGF-mediated axon branching in vitro and the increased apoptosis in Wnt5a $a^{-1-}$ SCG when neuronal survival is dependent on target-derived NGF, we examined innervation of distal sympathetic targets in $W n t 5 a^{-1-}$ mice. TH staining of SCG targets indicated far less sympathetic fibers innervate the nasal epithelium at E15.5 (Fig. 6P,Q) and the eye at E16.5 (Fig. $6 R, S)$ in $W n t 5 a^{-1-}$ embryos compared with wild type. These results indicate that $W n t 5 a^{-1-}$ sympathetic fibers fail to effectively innervate distal targets, before observed increases in neuronal apoptosis and cell loss at E17.5.

Because of the shortened anterior-posterior axis in Wnt5a $a^{-1-}$ embryos, we investigated the spatial organization of the entire sympathetic chain. By wholemount tyrosine hydroxylase staining, we determined that E16.5 Wnt5a ${ }^{-1-}$ embryos had aberrant segmentation and organization of the sympathetic chain that was more pronounced caudally (Fig. $6 U$, black arrowheads). These defects in segmentation were also observed in the nascent sympathetic chain at E13.5 (data not shown). Although we sometimes observed axonal projections emanating from $\mathrm{mu}-$ tant sympathetic ganglia at aberrant locations (Fig. 6U, white arrowhead) and appearing to be more disorganized, the proximal projections were often seen coursing along the intercostal arteries similar to that in wild-type embryos (Fig. 6T, $U$, arrows). The structural deficits observed in Wnt5a $a^{-1-}$ sympathetic chains is likely attributable to disruptions in elongation of the 
A

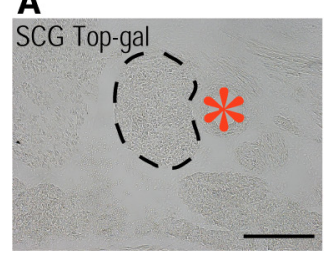

B
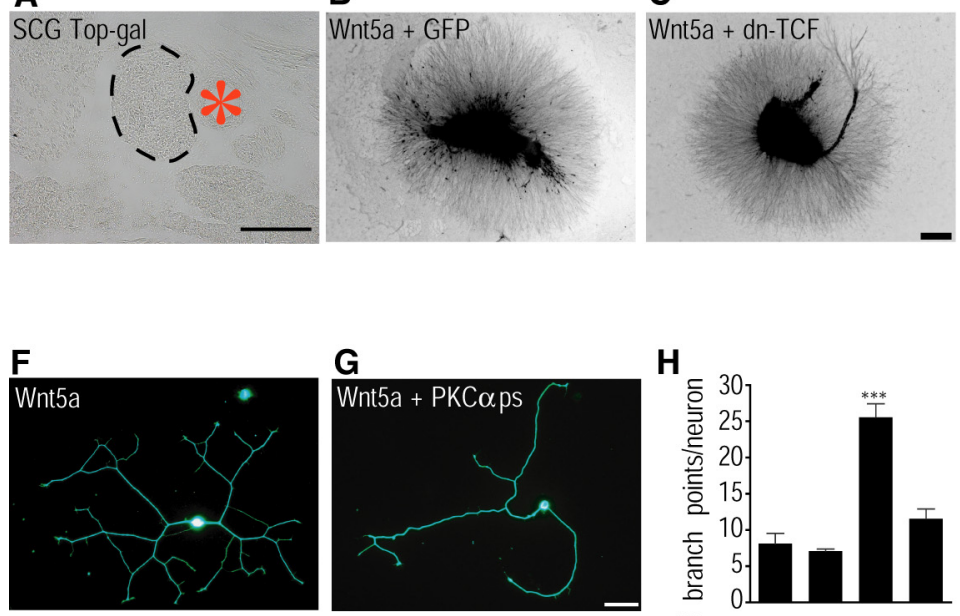

H

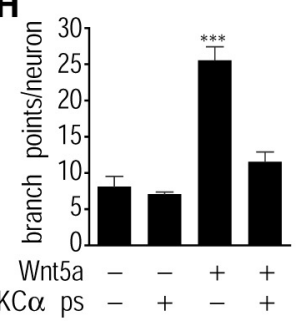

D

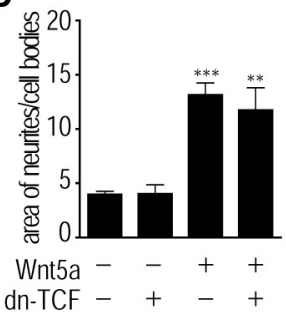

E

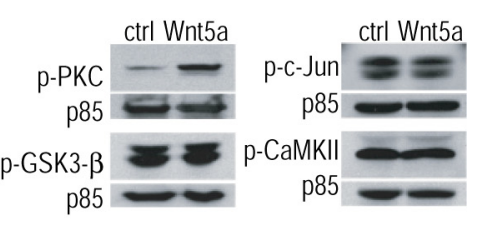

I

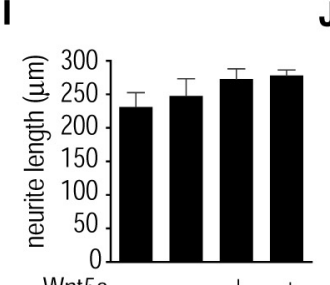

Wnt5a

$\mathrm{PKC} \alpha \mathrm{ps}-+-+$

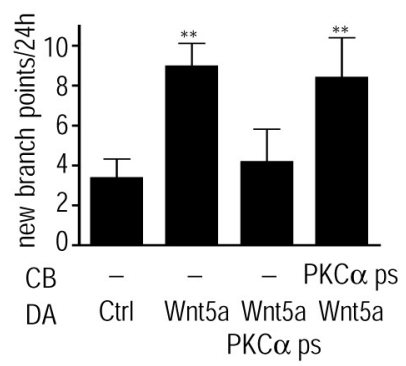

Figure 7. Wnt5a mediates branching via protein kinase C. $A$, Canonical Wnt/ $\beta$-catenin signaling in TOPGAL reporter transgenic mouse line is not detected in E16.5 SCG (outlined with dashed line); the asterisk (*) identifies a neighboring blood vessel. Scale bar, $100 \mu \mathrm{m}$. B, C, TCF transcriptional activity is not required for Wnt5a-mediated axon outgrowth. SCG explants infected with GFP (B) or dominant-negative-TCF (dn-TCF) (C) adenovirus show robust neurite outgrowth in response to Wnt5a. Ganglia were immunostained for $\beta$-III-tubulin. Scale bar, $320 \mu \mathrm{m}$. $\boldsymbol{D}, \mathbf{Q}$ Quantification of neurite outgrowth from SCG explants infected with either GFP or dn-TCF in the presence or absence of Wnt5a-containing media. Values are the mean \pm SEM for four explants. No significant difference in neurite outgrowth was observed between Wnt5a-treated explants infected with either GFP or dn-TCF adenovirus. Wnt5a-treated explants infected with GFP or dn-TCF were significantly different from control conditioned media-treated explants; ${ }^{* *} p<0.01$ and ${ }^{* * *} p<0.001$, as determined by one-way ANOVA followed by Tukey's multiple-comparisons test. $\boldsymbol{E}$, Cultured sympathetic neurons stimulated with recombinant Wnt5a (200 ng/ml; $30 \mathrm{~min})$ show increased levels of phosphorylated PKC (p-PKC). In contrast, levels of p-GSK3 $\beta$, p-C-Jun, p-CaMKII, were unaffected. All Western blots were stripped and reprobed for the p85 subunit of PI3-K as a loading control. $\boldsymbol{F}, \mathbf{G}$, Wnt5a-dependent axonal branching in sympathetic neurons requires PKC activity. Sympathetic neurons cultured for $24 \mathrm{~h}$ in the presence of Wnt5a show extensive branching $(\boldsymbol{F})$ that is abrogated by treatment with a myristoylated PKC $\alpha$ pseudosubstrate $(\boldsymbol{G})$. Neurons were immunostained for $\beta$-III-tubulin. Scale bar, $50 \mu \mathrm{m}$. $\boldsymbol{H}, \boldsymbol{I}$, Quantification of branch points per neuron $(\boldsymbol{H})$ and neurite length $(\boldsymbol{I})$ in low-density dissociated cultures treated with control or Wnt5a conditioned media, in the presence or absence of PKC $\alpha$-pseudosubstrate. Values are the mean \pm SEM from three independent experiments. ${ }^{* * *} p<0.001$ significantly different from control conditioned media-treated neurons, as determined by one-way ANOVA followed by Tukey's multiple-comparisons test.J, Quantification of new branch points per projection per $24 \mathrm{~h}$ in low-density compartmentalized cultures treated with control or Wnt5a-containing media exclusively on distal axons (DA), in the absence or presence of PKC $\alpha$-pseudosubstrate added to DA or cell bodies compartments (CB). Values are the mean \pm SEM from three independent experiments. ${ }^{* *} p<0.01$, significantly different from control conditioned media-treated neurons, as determined by one-way ANOVA followed by Tukey's multiple-comparisons test. PKC $\alpha$ (DA) is significantly different from PKC $\alpha(C B), p<0.05$.

anterior-posterior axis and integrity of the adjacent somites in early embryonic development (Goldstein and Kalcheim, 1991; Yamaguchi et al., 1999). Together, these results suggest that, although reduced innervation of targets might be the result of deficits in the intrinsic abilities of Wnt5a $a^{-1-}$ axons to branch and grow as seen in vitro, aberrant sympathetic chain formation and navigational errors en route to distal targets could also potentially contribute to the abnormalities in final target innervation observed in vivo.

\section{Wnt5a-mediated axonal branching requires $\mathrm{PKC}$ activity locally in axons}

Wnts signal through three major pathways: the canonical $\beta$-catenin-dependent pathway, the planar cell polarity (PCP) pathway, and a $\mathrm{Ca}^{2+}$-dependent pathway (Ciani and Salinas, $2005)$. Activation of the canonical Wnt signaling pathway stabilizes the cytoplasmic effector, $\beta$-catenin, which then translocates to the nucleus and binds to the transcription factors T-cell factor (TCF) and lymphoid enhancer factor (LEF) to activate the transcription of target genes. Canonical Wnt pathway activity was assessed in the developing SCG at E16.5, using TOPGAL transgenic mice in which $L a c Z$ expression reports $\beta$-catenin transcriptional activity (DasGupta and Fuchs, 1999). The SCG did not show any $\mathrm{X}$-gal staining for $\beta$-galactosidase (Fig. $7 A$ ), whereas prominent staining was seen in other tissues such as the cochlea (supplemental Fig. 7, available at www.jneurosci.org as supplemental material) as previously reported (DasGupta and Fuchs,
1999). Interfering with the canonical Wnt signaling pathway by expression of a dominant-negative inhibitor of the transcription factor TCF in SCG explants had no effect on Wnt5a-dependent axonal outgrowth (Fig. $7 B-D$ ). It must be noted here that $\beta$-catenin also signals via a transcription-independent mechanism by complexing with cadherins and regulating cell adhesion (Nelson and Nusse, 2004). Although we show that Wnt5a responses in sympathetic neurons are independent of $\beta$-cateninmediated transcriptional activity, we cannot completely exclude the role of $\beta$-catenin in mediating Wnt5a responses.

Noncanonical Wnt signaling has been shown to elicit an increase in intracellular $\mathrm{Ca}^{2+}$ and activate $\mathrm{Ca}^{2+}$-sensitive enzymes such as CaMKII and PKC (Wodarz and Nusse, 1998; Veeman et al., 2003; Kohn and Moon, 2005). In the Wnt-PCP pathway, noncanonical Wnts activate the small GTPases Rho and Rac as well as c-Jun N-terminal kinase (JNK) to regulate the uniform orientation of cells within an epithelial plane, cellular migration, and convergent-extension movements during neurulation (Montcouquiol et al., 2006). To investigate the signaling pathways by which Wnt5a influences axonal branching, we used a battery of phosphospecific antibodies to identify the signaling effectors activated by Wnt5a in cultured sympathetic neurons. Cultured sympathetic neurons stimulated with recombinant Wnt5a (200 ng/ml; $30 \mathrm{~min}$ ) showed a significant increase in levels of phosphorylated PKC, using a pan-phospho-PKC antibody that recognizes endogenous $\mathrm{PKC} \alpha, \beta \mathrm{I}, \beta \mathrm{II}, \delta, \varepsilon, \eta$, and $\theta$ isoforms, when phosphorylated on Ser660 (Fig. 7E). Levels of phos- 
phorylated GSK-3 $\beta$, a component in the canonical Wnt signaling pathway, phosphorylated c-jun, a component of the JNK signaling pathway, and phosphorylated CaMKII were unaffected by Wnt5a treatment (Fig. 7E). Additionally, Wnt5a treatment did not elicit any changes in the two major signaling pathways known to be activated by NGF, as evidenced by equivalent levels of phosphorylated Akt and Erk1/2 in control and Wnt5a-treated neurons (supplemental Fig. 6, available at www.jneurosci.org as supplemental material).

Given the Wnt5a-induced increase in phosphorylated PKC, we asked whether PKC is required for Wnt5a-mediated axonal branching in sympathetic neurons. To inhibit PKC activity, we used myristoylated PKC $\alpha$ pseudosubstrate $(25 \mu \mathrm{M})$, a cellpermeable peptide that blocks PKC activity by preventing substrate binding (Eichholtz et al., 1993; Wolf et al., 2008). PKC inhibition effectively repressed Wnt5a-induced branching (Fig. $7 F-H)$ without affecting neurite length in control and Wnt5atreated neurons (Fig. 7I). Furthermore, by spatially inhibiting PKC activity in the distal axons or cell bodies of compartmentalized cultures, we found that Wnt5a-induced axon branching requires local PKC signaling in distal axons (Fig. 7J). Together, our results indicate that Wnt5a-mediated axon remodeling in sympathetic neurons requires PKC activity locally in axons.

\section{Discussion}

The family of neurotrophins provides one of the best examples of target-derived instructive cues that regulate multiple facets of neuronal development including cell survival, axonal and dendritic growth, and synapse formation. Our study highlights a previously uncharacterized regulatory pathway in which targetderived NGF promotes axonal branching and target innervation during sympathetic neuron development by enhancing neuronal expression of Wnt5a. We found that Wnt5a is highly expressed in sympathetic neurons at a developmental time when their axons are reaching and innervating final target tissues. Wnt5a expression is enhanced by NGF, but not by NT-3, an intermediate target-derived axon growth factor for sympathetic neurons. Wnt5a exerts a rapid effect on promoting axon branching and a more delayed effect on enhancing extension. Sympathetic neurons derived from $W n t 5 a^{-1-}$ mice show deficits in NGFdependent axonal branching and growth, but not survival, in vitro. In the absence of $W n t 5 a$, sympathetic innervation of final target tissues is absent or highly attenuated at early stages preceding increased neuronal apoptosis. These findings reveal Wnt5a as a novel NGF effector that regulates axonal growth, branching, and innervation of sympathetic target tissues. Our study adds to emerging evidence that mechanisms of cross talk between targetderived neurotrophins and growth factors expressed in neurons could underlie neurotrophin actions in the nervous system. In a recent study, autocrine signaling of glucocorticoid-induced tumor necrosis factor receptor-related protein (GITR) and its ligand GITRL within developing sympathetic neurons was shown to be essential for target-derived NGF to promote axon growth and target innervation (O'Keeffe et al., 2008).

Our results indicate that the aspect of NGF-dependent growth most sensitive to Wnt5a signaling is axonal branching. In the presence of NGF, sympathetic neurons lacking Wnt5a show significantly reduced axonal branching both in vitro and in vivo. Within minutes of Wnt5a treatment, axons of sympathetic neurons show distinct branching of growth cones, sprouting of processes from the growth cones, and extension of these new branches. Within $2 \mathrm{~h}$ of Wnt5a addition, axons develop complex arborization patterns, whereas a significant effect on axon exten- sion was only observed after extended periods of growth (3d) in the presence of the Wnt ligand. In vivo, an acute effect of Wnt5a in sympathetic axons might be to initiate axonal branching on axons reaching final targets, whereas a delayed effect of Wnt5a might be to promote extension of the newly formed branches to completely cover the target territory.

We demonstrated that $W n t 5 a^{-1-}$ neurons show profound deficits in axon branching and growth in vitro. There is no cellautonomous requirement for Wnt5a in mediating neuronal survival, and yet enhanced cell death in vivo is observed in $W n t 5 a^{-1-}$ SCGs at times when axons are actively innervating target tissues. There are no statistically significant differences in SCG cell number between wild-type and $W n t 5 a^{-1-}$ embryos at E13.5-E15.5, before, or at very early stages of, target innervation. Since most neurogenesis in mouse SCG is complete by E15.5 and the major period of gliogenesis occurs postnatally (Fagan et al., 1996), the normal SCG cell number at E15.5 suggests that proliferation and survival of neuronal precursors are unaffected by the absence of Wnt5a. However, at E17.5, there is a significant increase in cell loss and apoptosis, similar to the phenotype observed in $N G F^{-1-}$ and $\operatorname{Trk} A^{-l-}$ mice, suggesting that, in the absence of $W n t 5 a$, axons are unable to reach final targets and gain access to NGF. In support of this hypothesis, distal target tissues such as the nasal epithelium and eye show far less sympathetic fibers at the earliest stages (E15.5-E16.5) of final target innervation, preceding significant cell loss. In light of our in vitro data showing that Wnt5a is required in dissociated sympathetic neurons for NGF-mediated axon branching and growth, we propose that the most parsimonious explanation for reduced innervation seen in vivo is an intrinsic inability of $W n t 5 a^{-1-}$ axons to grow and branch into final targets. We also observed deficits in sympathetic chain segmentation in Wnt5a-deficient embryos. Segmentation defects may arise, in part, from the reported disruptions in the rostrocaudal integrity of the adjacent somites in the embryonic Wnt5a-null mice (Yamaguchi et al., 1999). The rostral somites are normal in E9.5 Wnt5a mutants, whereas the caudal somites become progressively smaller in size (Yamaguchi et al., 1999). We observed a similar rostral-caudal decline in the integrity of the sympathetic chain ganglia in $W n t 5 a^{-1-}$ embryos. Thus, the SCG, the rostralmost ganglia in the sympathetic chain, appeared to be normally located at the bifurcation of the carotid artery, and of normal size until E15.5, and it seems less likely that the reduced innervation of distal SCG targets arises primarily from disruptions in the sympathetic chain. However, we cannot completely exclude the possibilities that defects in somite integrity in Wnt5a $a^{-1-}$ embryos and subsequent effects on sympathetic chain segmentation or non-cell-autonomous roles for Wnt5a in intermediate target tissues lead to navigational deficits that contribute, at least in part, to decreased final target innervation.

The localized effects of Wnt5a in axons combined with the result that Wnt5a-dependent axon branching is transcription independent suggest a direct modulation of the actin-microtubule cytoskeleton in axons. Recently, Wnt3a was shown to mediate rapid changes in microtubule organization and directionality in developing DRG neurons, promoting growth cone pausing, expansion, and branching (Purro et al., 2008). Our results indicate that regulation of classical PKCs that are sensitive to elevations in intracellular $\mathrm{Ca}^{2+}$ and diacylglycerol might underlie the actions of Wnt5a in sympathetic axons. Interestingly, Wnt5a induces extensive remodeling of the actin cytoskeletal architecture to promote directional motility and invasiveness of melanoma cells by a $\beta$-catenin-independent mechanism that involves phosphorylation and activation of classical PKCs (Weeraratna et al., 2002; 
Witze et al., 2008). Given the ability of PKCs to activate multiple signaling effectors including known regulators of axon morphology such as FAK (focal adhesion kinase) (Cohen et al., 2002; Rico et al., 2004) and GAP-43 (Aigner et al., 1995; He et al., 1997), additional studies are required to shed light on local PKC effectors in axons that contribute to axonal branching. However, consistent with our observations of a local requirement for PKC activity in sympathetic axons in regulating branching, a previous study in compartmentalized cultures demonstrated that NGFdependent axon growth is attenuated by inhibition of PKC activity in distal neurites but not cell bodies (Campenot et al., 1994).

Although much is known about Wnt signaling, our current knowledge of the regulation of Wnt genes themselves is very limited. We propose that NGF secreted by target tissues signals retrogradely to sympathetic nuclei to ensure precise spatial and temporal control of Wnt5a expression during target innervation. In vivo studies indicate that retrograde NGF signaling activates transcriptional programs essential for long-term changes in axon growth through the nuclear effectors, cyclic adenosine monophosphate element-binding protein (CREB), nuclear factor of activated T-cells (NFAT), and serum response factor (SRF) (Lonze and Ginty, 2002; Graef et al., 2003; Wickramasinghe et al., 2008). Conditional deletion of SRF in NGF-responsive sensory neurons revealed a specific requirement for SRF in axonal arborization during the final phase of target innervation (Wickramasinghe et al., 2008). Interestingly, a consensus SRF binding site, the CArG-like sequence is present in a $1 \mathrm{~kb}$ region upstream of the transcriptional start site of $W n t 5 a$. Expression of a dominant-negative form of SRF, but not dominant-negative inhibitors of CREB and NFAT, substantially attenuated Wnt5a levels in cultured sympathetic neurons maintained in the presence of NGF (D. Bodmer and R. Kuruvilla, unpublished observations). Together, these results allude to an SRF-dependent mechanism of $W n t 5 a$ expression in developing sympathetic neurons, although additional studies are warranted to test this hypothesis.

Our results demonstrate a critical role for Wnt5a signaling within sympathetic neurons to mediate branching; however, this does not preclude an additive role for Wnts secreted by target tissues themselves in mediating terminal arborization. RT-PCR analyses showed that Wnt5a is present in the SCG target tissues, salivary glands and trachea (data not shown). In a recent study, BDNF signaling in cerebellar granule cell precursors was shown to cooperate with exogenous BDNF to amplify a local BDNF gradient and thus direct migration of cerebellar granule cell precursors (Zhou et al., 2007). In an analogous manner, Wnt5a secreted by sympathetic axons might act in concert with targetderived Wnt5a to amplify axonal responses within target tissues.

Our study raises the possibility that other neurotrophindependent processes in the developing nervous system might be mediated in a similar manner through a hierarchical trophic factor cascade. In particular, both neurotrophins and Wnts have been shown to be potent synaptogenic factors (Vicario-Abejón et al., 2002; Speese and Budnik, 2007); it remains to be determined whether neurotrophins promote synapse formation by regulating the expression and signaling of Wnts. Uncovering mechanisms of cross talk between neurotrophins and other signaling pathways will provide new insight into how a relatively small number of growth factors can be used to assemble complex, yet precise, neuronal circuits during development.

\section{References}

Aigner L, Arber S, Kapfhammer JP, Laux T, Schneider C, Botteri F, Brenner HR, Caroni P (1995) Overexpression of the neural growth-associated protein GAP-43 induces nerve sprouting in the adult nervous system of transgenic mice. Cell 83:269-278.

Albers KM, Wright DE, Davis BM (1994) Overexpression of nerve growth factor in epidermis of transgenic mice causes hypertrophy of the peripheral nervous system. J Neurosci 14:1422-1432.

Belliveau DJ, Krivko I, Kohn J, Lachance C, Pozniak C, Rusakov D, Kaplan D, Miller FD (1997) NGF and neurotrophin-3 both activate TrkA on sympathetic neurons but differentially regulate survival and neuritogenesis. J Cell Biol 136:375-388.

Brennan C, Rivas-Plata K, Landis SC (1999) The p75 neurotrophin receptor influences NT-3 responsiveness of sympathetic neurons in vivo. Nat Neurosci 2:699-705.

Campenot RB (1977) Local control of neurite development by nerve growth factor. Proc Natl Acad Sci U S A 74:4516-4519.

Campenot RB (1979) Independent control of local environment of somas and neurites. Methods Enzymol 58:302-307.

Campenot RB, Draker DD, Senger DL (1994) Evidence that protein kinase $\mathrm{C}$ activities involved in regulating neurite growth are localized to distal neurites. J Neurochem 63:868-878.

Causing CG, Gloster A, Aloyz R, Bamji SX, Chang E, Fawcett J, Kuchel G, Miller FD (1997) Synaptic innervation density is regulated by neuronderived BDNF. Neuron 18:257-267.

Chen X, Ye H, Kuruvilla R, Ramanan N, Scangos KW, Zhang C, Johnson NM, England PM, Shokat KM, Ginty DD (2005) A chemical-genetic approach to studying neurotrophin signaling. Neuron 46:13-21.

Ciani L, Salinas PC (2005) WNTs in the vertebrate nervous system: from patterning to neuronal connectivity. Nat Rev Neurosci 6:351-362.

Cohen ED, Mariol MC, Wallace RM, Weyers J, Kamberov YG, Pradel J, Wilder EL (2002) DWnt4 regulates cell movement and focal adhesion kinase during Drosophila ovarian morphogenesis. Dev Cell 2:437-448.

DasGupta R, Fuchs E (1999) Multiple roles for activated LEF/TCF transcription complexes during hair follicle development and differentiation. Development 126:4557-4568.

Diamond J, Holmes M, Coughlin M (1992) Endogenous NGF and nerve impulses regulate the collateral sprouting of sensory axons in the skin of the adult rat. J Neurosci 12:1454-1466.

Edwards RH, Rutter WJ, Hanahan D (1989) Directed expression of NGF to pancreatic beta cells in transgenic mice leads to selective hyperinnervation of the islets. Cell 58:161-170.

Eichholtz T, de Bont DB, de Widt J, Liskamp RM, Ploegh HL (1993) A myristoylated pseudosubstrate peptide, a novel protein kinase $\mathrm{C}$ inhibitor. J Biol Chem 268:1982-1986.

Fagan AM, Zhang H, Landis S, Smeyne RJ, Silos-Santiago I, Barbacid M (1996) TrkA, but not TrkC, receptors are essential for survival of sympathetic neurons in vivo. J Neurosci 16:6208-6218.

Francis N, Farinas I, Brennan C, Rivas-Plata K, Backus C, Reichardt L, Landis S (1999) NT-3, like NGF, is required for survival of sympathetic neurons, but not their precursors. Dev Biol 210:411-427.

Franklin JL, Johnson EM (1998) Control of neuronal size homeostasis by trophic factor-mediated coupling of protein degradation to protein synthesis. J Cell Biol 142:1313-1324.

Gallo G, Letourneau PC (1998) Localized sources of neurotrophins initiate axon collateral sprouting. J Neurosci 18:5403-5414.

Glebova NO, Ginty DD (2004) Heterogeneous requirement of NGF for sympathetic target innervation in vivo. J Neurosci 24:743-751.

Goldstein RS, Kalcheim C (1991) Normal segmentation and size of the primary sympathetic ganglia depend upon the alternation of rostrocaudal properties of the somites. Development 112:327-334.

Graef IA, Wang F, Charron F, Chen L, Neilson J, Tessier-Lavigne M, Crabtree GR (2003) Neurotrophins and netrins require calcineurin/NFAT signaling to stimulate outgrowth of embryonic axons. Cell 113:657-670.

Hall AC, Lucas FR, Salinas PC (2000) Axonal remodeling and synaptic differentiation in the cerebellum is regulated by WNT-7a signaling. Cell 100:525-535.

Hassankhani A, Steinhelper ME, Soonpaa MH, Katz EB, Taylor DA, Andrade-Rozental A, Factor SM, Steinberg JJ, Field LJ, Federoff HJ (1995) Overexpression of NGF within the heart of transgenic mice causes hyperinnervation, cardiac enlargement, and hyperplasia of ectopic cells. Dev Biol 169:309-321.

He Q, Dent EW, Meiri KF (1997) Modulation of actin filament behavior by GAP-43 (neuromodulin) is dependent on the phosphorylation status of serine 41, the protein kinase C site. J Neurosci 17:3515-3524. 
Kohn AD, Moon RT (2005) Wnt and calcium signaling: beta-cateninindependent pathways. Cell Calcium 38:439-446.

Krylova O, Herreros J, Cleverley KE, Ehler E, Henriquez JP, Hughes SM, Salinas PC (2002) WNT-3, expressed by motoneurons, regulates terminal arborization of neurotrophin-3-responsive spinal sensory neurons. Neuron 35:1043-1056.

Kuruvilla R, Zweifel LS, Glebova NO, Lonze BE, Valdez G, Ye H, Ginty DD (2004) A neurotrophin signaling cascade coordinates sympathetic neuron development through differential control of TrkA trafficking and retrograde signaling. Cell 118:243-255.

Lonze BE, Ginty DD (2002) Function and regulation of CREB family transcription factors in the nervous system. Neuron 35:605-623.

Lucas FR, Salinas PC (1997) WNT-7a induces axonal remodeling and increases synapsin I levels in cerebellar neurons. Dev Biol 192:31-44.

Markus A, Patel TD, Snider WD (2002) Neurotrophic factors and axonal growth. Curr Opin Neurobiol 12:523-531.

Montcouquiol M, Crenshaw EB 3rd, Kelley MW (2006) Noncanonical Wnt signaling and neural polarity. Annu Rev Neurosci 29:363-386.

Murdoch B, Chadwick K, Martin M, Shojaei F, Shah KV, Gallacher L, Moon RT, Bhatia M (2003) Wnt-5A augments repopulating capacity and primitive hematopoietic development of human blood stem cells in vivo. Proc Natl Acad Sci U S A 100:3422-3427.

Nelson WJ, Nusse R (2004) Convergence of Wnt, beta-catenin, and cadherin pathways. Science 303:1483-1487.

O’Keeffe GW, Gutierrez H, Pandolfi PP, Riccardi C, Davies AM (2008) NGF-promoted axon growth and target innervation requires GITRLGITR signaling. Nat Neurosci 11:135-142.

Patel TD, Jackman A, Rice FL, Kucera J, Snider WD (2000) Development of sensory neurons in the absence of NGF/TrkA signaling in vivo. Neuron 25:345-357.

Purro SA, Ciani L, Hoyos-Flight M, Stamatakou E, Siomou E, Salinas PC (2008) Wnt regulates axon behavior through changes in microtubule growth directionality: a new role for adenomatous polyposis coli. J Neurosci 28:8644-8654.

Rico B, Beggs HE, Schahin-Reed D, Kimes N, Schmidt A, Reichardt LF (2004) Control of axonal branching and synapse formation by focal adhesion kinase. Nat Neurosci 7:1059-1069.
Rubin E (1985) Development of the rat superior cervical ganglion: ganglion cell maturation. J Neurosci 5:673-684.

Speese SD, Budnik V (2007) Wnts: up-and-coming at the synapse. Trends Neurosci 30:268-275.

Veeman MT, Axelrod JD, Moon RT (2003) A second canon. Functions and mechanisms of beta-catenin-independent Wnt signaling. Dev Cell 5:367-377.

Vicario-Abejón C, Owens D, McKay R, Segal M (2002) Role of neurotrophins in central synapse formation and stabilization. Nat Rev Neurosci 3:965-974.

Weeraratna AT, Jiang Y, Hostetter G, Rosenblatt K, Duray P, Bittner M, Trent JM (2002) Wnt5a signaling directly affects cell motility and invasion of metastatic melanoma. Cancer Cell 1:279-288.

Wickramasinghe SR, Alvania RS, Ramanan N, Wood JN, Mandai K, Ginty DD (2008) Serum response factor mediates NGF-dependent target innervation by embryonic DRG sensory neurons. Neuron 58:532-545.

Witze ES, Litman ES, Argast GM, Moon RT, Ahn NG (2008) Wnt5a control of cell polarity and directional movement by polarized redistribution of adhesion receptors. Science 320:365-369.

Wodarz A, Nusse R (1998) Mechanisms of Wnt signaling in development. Annu Rev Cell Dev Biol 14:59-88.

Wolf AM, Lyuksyutova AI, Fenstermaker AG, Shafer B, Lo CG, Zou Y (2008) Phosphatidylinositol-3-kinase-atypical protein kinase $\mathrm{C}$ signaling is required for Wnt attraction and anterior-posterior axon guidance. J Neurosci 28:3456-3467.

Yamaguchi TP, Bradley A, McMahon AP, Jones S (1999) A Wnt5a pathway underlies outgrowth of multiple structures in the vertebrate embryo. Development 126:1211-1223.

Ye H, Kuruvilla R, Zweifel LS, Ginty DD (2003) Evidence in support of signaling endosome-based retrograde survival of sympathetic neurons. Neuron 39:57-68.

Zhou P, Porcionatto M, Pilapil M, Chen Y, Choi Y, Tolias KF, Bikoff JB, Hong EJ, Greenberg ME, Segal RA (2007) Polarized signaling endosomes coordinate BDNF-induced chemotaxis of cerebellar precursors. Neuron 55: 53-68. 